\title{
Les souvenirs d'anciens élèves, de la Renaissance à la fin de l'ancien régime scolaire. Esquisse pour une analyse historienne
}

The memories of former students from the Renaissance to the end of the

educational old regime. Propositions for an historical analysis

Pierre Caspard

\section{(2) OpenEdition}

Journals

Édition électronique

URL : https://journals.openedition.org/histoire-education/4388

DOI : 10.4000/histoire-education.4388

ISSN : 2102-5452

Éditeur

ENS Éditions

Édition imprimée

Date de publication : 15 janvier 2019

Pagination : 27-61

ISBN : 979-10-362-0144-8

ISSN : 0221-6280

Référence électronique

Pierre Caspard, «Les souvenirs d'anciens élèves, de la Renaissance à la fin de l'ancien régime scolaire. Esquisse pour une analyse historienne », Histoire de l'éducation [En ligne], 151 | 2019, mis en ligne le 01 janvier 2022, consulté le 07 janvier 2022. URL : http://journals.openedition.org/histoire-education/ 4388 ; DOI : https://doi.org/10.4000/histoire-education.4388 


\section{Les souvenirs d'anciens élèves, de la Renaissance à la fin de l'ancien régime scolaire. Esquisse pour une analyse historienne}

\section{Pierre Caspard}

Les souvenirs d'anciens élèves ont la rareté et la discontinuité d'une source erratique, qui dissuade les historiens de l'école et des apprentissages d'y recourir autrement que pour illustrer, étayer ou nuancer l'analyse qu'ils font des sources institutionnelles, normatives ou statistiques. C'est dans cette perspective que les historiens font le plus généralement contribuer les souvenirs d'élèves à l'histoire de l'école, de l'éducation, de la famille et de l'enfance. Nous y reviendrons.

De leur côté, les historiens de la littérature, de la genèse et de la poétique des œuvres, ont accordé une attention spécifique à deux types de souvenirs : les mémoires et les autobiographies. Ils ont même circonscrit un sous-genre, le récit d'enfance, peu présent dans les premiers, plus développé dans les seconds, parfois devenu autonome aux XIXe et XX ${ }^{e}$ siècles. Mais qu'ils apparaissent dans l'un ou dans l'autre de ces genres, les souvenirs d'anciens élèves ont suscité chez eux un intérêt limité. C'est que, pour celui qui étudie des œuvres, l'élève se prête infiniment moins que l'enfant aux commentaires et analyses qui privilégient les "récits fondateurs" d'une personnalité, généralement celle de grands auteurs, et qu'il se prête également moins à des mises en forme littéraire. Les souvenirs d'élèves semblent en effet offrir "peu de variétés dans les solutions narratives", se réduisant parfois à "un répertoire fastidieux et stéréotypé des étapes d'une scolarité " ${ }^{1}$ parmi un nombre restreint d'institutions : Famille, Préceptorat, Couvent, Collège, École, Lycée, supposées déjà bien connues par ailleurs, ès qualités.

Les souvenirs d'élèves sont pourtant abordés de façon oblique par les historiens de la littérature. L'édition critique des souvenirs de grands écrivains

1 Emmanuèle Lesne-Jaffro, «Le récit d'enfance à la première personne au XVIIe siècle : entre mémoire et fiction", in Anne Chevalier, Carole Dornier (dir.), Le Récit d'enfance et ses modèles, Caen, université de Caen, 2003, p. 126. 
ou mémorialistes les conduit à confronter le texte de leurs souvenirs avec la matérialité des faits et personnages qu'ils évoquent, et à faire le départ, dans les récits d'apprentissage, entre vérité et fiction ${ }^{2}$. Ils peuvent également analyser les différentes mises en forme qu'ont pu faire de leurs souvenirs d'élèves et d'apprentissage quelques grands auteurs, pour y distinguer la part du discours et du récit, de la poétique et de l'événementiel ou du factuel. Ceci vaut essentiellement à partir du $\mathrm{XIX}^{\mathrm{e}}$ siècle, où la popularité naissante du souvenir et du récit d'enfance et la place qu'y prend le souci de soi conduit des professionnels de l'écriture littéraire à y revenir à plusieurs reprises, sous différentes formes et à différents moments de leur vie ${ }^{3}$.

Les souvenirs d'anciens élèves devenus hommes et femmes de lettres, le plus souvent mis à contribution par les historiens de l'enseignement, justifient donc une interrogation particulière sur leur régime de vérité. Mais Montaigne ou Agrippa d'Aubigné, George Sand ou Flaubert ne sont pas seuls à avoir évoqué des souvenirs d'élèves. De Thomas Platter, professeur de grec (né en 1499) à André-Jacob Roubo, menuisier-ébéniste (1739), Jean Conan, tisserand (1765), Louis-Philippe, roi des Français (1773) ou Camille Flammarion, astronome (1842), d'Alix Le Clerc, religieuse (1576) à Herculine Barbin, enseignant(e) (1838), des centaines d'hommes et de femmes de toutes conditions ont évoqué leurs études, leur scolarité et leurs apprentissages.

Nous avons donc repéré l'ensemble de leurs souvenirs, sans les segmenter a priori selon le milieu social, l'activité ou le sexe des scripteurs, ni les instances ou acteurs de leurs apprentissages, ni aucun critère autre que l'existence même de souvenirs d'élèves dans leurs mémoires. La période d'observation retenue va des premières apparitions récurrentes de souvenirs d'élèves, à la Renaissance, jusqu'aux décennies médianes du $\mathrm{XIX}^{\mathrm{e}}$ siècle, que nous définissons comme la fin de l'ancien régime scolaire, c'est-à-dire comme le passage progressif

2 Il suffirait d'évoquer toutes les éditions critiques des œuvres d'écrivains rapportant des souvenirs d'enfance, d'école et d'études, publiés dans la Bibliothèque de la Pléiade (Gallimard), par exemple ceux de J.-J. Rousseau, N. Restif de la Bretonne, B. Constant, F.-R. de Chateaubriand, Stendhal, G. Sand, G. Flaubert, J. Vallès, J.-P. Sartre, S. de Beauvoir, etc. Les nombreuses études spécifiquement consacrées à l'enfance et la jeunesse d'écrivains peuvent également aborder de façon critique le récit qu'ils ont fait de leurs expériences d'élèves.

3 On peut ainsi confronter les souvenirs d'élève de Jules Vallès dans le Testament d'un blagueur (1869), L'Enfant (1878), Le Bachelier (1881) et les Souvenirs d'un étudiant pauvre, ces derniers étant sous-titrés : Mémoires vrais (1884). 
d'un dispositif scolaire, résultant d'initiatives anciennes et diverses, privées et publiques, à un système scolaire, désormais largement organisé par l'État ${ }^{4}$.

Le repérage de l'ensemble de ces souvenirs conduit à une première réflexion sur le genre même des textes où leurs auteurs les ont consignés.

\section{Les souvenirs d'élèves, au carrefour de genres}

Les souvenirs d'anciens élèves se donnent à connaître dans des textes qui ressortissent à des genres différents, présentant entre eux, dans la longue durée, glissements et chevauchements.

\section{Mémoires}

Les mémoires, dans la définition stricte du terme, se caractérisent par le souci des auteurs de témoigner sur le cours d'une Histoire dont ils ont été personnellement les acteurs et les témoins. Né à la fin du Moyen Âge, le genre des mémoires fleurit aux XVI ${ }^{\mathrm{e}}$ et XVII ${ }^{\mathrm{e}}$ siècles, connaît un immense succès public au lendemain de la Révolution et de l'Empire, pour n'être ensuite illustré que

4 La grande majorité des mémoires et autobiographies publiés en France sont conservés dans la série Ln27 de la Bibliothèque nationale de France. Nous avons consulté (octobre 2014) le site "Les écrits du for privé" qui recense ceux qui ont été rédigés et publiés, plus ou moins longtemps après leur rédaction, par 51 auteurs nés au XVI ${ }^{\mathrm{e}}$ siècle, $96 \mathrm{au} \mathrm{XVII}{ }^{\mathrm{e}}, 490 \mathrm{au}$ XVIII ${ }^{\mathrm{e}}, 217 \mathrm{au}$ XIX $^{\mathrm{e}}$ siècle, ces derniers pratiquement tous nés avant 1850. Des titres ont par ailleurs été repérés dans Alfred Fierro, Bibliographie critique des mémoires sur la Révolution, écrits ou traduits en français, Paris, Service des travaux historiques de la Ville de Paris, 1989 (1502 références); Jean Tulard (dir.), Nouvelle bibliographie critique des mémoires sur l'époque napoléonienne, écrits ou traduits en français, Genève, Droz, 1991 (1 527 références); Guillaume Bertier de Sauvigny, Alfred Fierro, Bibliographie critique des mémoires sur la Restauration, écrits ou traduits en français, Genève, Droz, 1988 (1019 références), Denis Bertholet, Les Français par eux-mêmes, 1815-1885, Paris, Olivier Orban, 1991 (358 références; les dates indiquées dans ce titre sont celles de la publication des mémoires) ainsi que dans de très nombreuses thèses, ouvrages, actes de colloques, numéros spéciaux de revues publiés sur les mémoires, les autobiographies, l'enfance, les femmes, la famille, l'éducation..., particulièrement depuis les années 1970 .

Nous avons consulté environ 1300 des titres relevés, pour en retenir, à ce jour, 619 qui contiennent des souvenirs, succincts ou circonstanciés, d'apprentissages, d'études et de scolarité. Même s'il vise idéalement à l'exhaustivité, le corpus ainsi constitué ne saurait prétendre y atteindre, soit que quelques titres pertinents se soient révélés difficiles d'accès, soit que d'autres aient échappé à notre attention, soit que des souvenirs restés inédits fassent un jour l'objet de publication, comme il arrive périodiquement. On peut cependant considérer que l'adjonction de nouveaux titres n'affectera que très marginalement les conclusions ici présentées. Par ailleurs, 99 des auteurs du corpus sont de nationalité étrangère mais ont écrit en français ou ont rapidement été traduits; ils sont principalement suisses (32 dont 27 romands), allemands (17), anglais (14) et italiens (14). En première approximation et sans aborder plus avant cette question, il apparait que les souvenirs d'élèves de tous les petits Occidentaux présentent un haut degré de familiarité. Par exemple, cf. infra les expériences préceptorales de trois fils d'officiers français, belge et suisse. 
plus ponctuellement ${ }^{5}$. En principe, l'évocation de l'enfance comme âge des apprentissages n'a pas sa place dans les mémoires, qui débutent logiquement avec l'"entrée dans le monde" de l'auteur. Ceux de Philippe de Commynes, né en 1447, débutent ainsi : "Au saillir de mon enfance et en l'âge de pouvoir monter à cheval, je fus amené à Lille devers le duc de Bourgogne, qui me prit à son service. Et fut l'an $1464 »^{6}$. Ses dix-sept premières années sont donc passées sous silence. Le duc de Choiseul, né en 1719, devenu ministre de Louis XV, informe ses lecteurs que "Mon enfance et ma jeunesse se sont passées comme celles de tout le monde" (!) et commence le récit de sa vie en $1738^{7}$. Les mémoires de François Guizot (1787-1874), dont le souci de l'éducation des autres aurait pu faire espérer quelques souvenirs et réflexions sur la sienne propre, débutent par : "Je ne suis entré qu'en 1814 dans la vie publique"

Pourtant, au fur et à mesure qu'on s'éloigne des origines médiévales du genre, certains mémorialistes n'hésitent pas à se mettre en scène en évoquant leurs ancêtres, leur entrée dans la vie et leur enfance, incluant tout ou partie des études et apprentissages, scolaires ou domestiques, dont ils ont bénéficié. C'est le cas d'Agrippa d'Aubigné (1552-1630), homme de guerre et poète ${ }^{9}$, de la reine Christine de Suède (1626-1689) ou du roi de Pologne Stanislas Auguste Poniatowski (1737-1798), ces deux derniers entremêlant - en français - l'histoire de leur pays à la leur ${ }^{10}$. Parfois, les pages sur l'enfance n'apparaissent

5 Les études sur les mémoires, la définition du genre, ses rapports et ses frontières avec l'autobiographie et le roman à la première personne, sont extrêmement abondantes. Le meilleur guide en est aujourd'hui Françoise Simonet-Tenant (dir.), avec la coll. de Michel Braud, Jean-louis Jeannelle, Philippe Lejeune et Véronique Montément, Dictionnaire de l'autobiographie. Écritures de soi de langue française, Paris, Champion, 2017. Notons qu'il ne contient aucune entrée aux mots "élève", "école", "instruction", "études " ou "apprentissages"; l'entrée "éducation" ne traite que de l'écriture autobiographique comme exercice scolaire contemporain.

6 Philippe de Commynes, Mémoires, Paris, Les Belles Lettres, 1964. Mais les apprentissages peuvent déborder largement sur les entrées dans le monde. Né en 1598, César de Choiseul du Plessis-Praslin, futur maréchal de France, en témoigne dans ses mémoires, écrits à la troisième personne : "Quand il eut quatorze ans, on lui donna un régiment d'infanterie, qu'il s'attacha aussitôt à commander, afin de s'en rendre promptement capable "...! Mémoires des divers emplois et des principales actions du Maréchal du Plessy, Paris, 1676. D’une manière générale, nous n'avons pas pris en compte les souvenirs d'apprentissages professionnels effectués sur le lieu et dans le cadre mêmes de l'exercice d'un métier ou d'une fonction, qu'il s'agisse de ceux d'officiers, de négociants, d'artisans ou de parlementaires.

7 Étienne-François, duc de Choiseul, Mémoires [1790], Paris, Plon, 1904.

8 François Guizot, Mémoires pour servir à l'histoire de mon temps, t. 1, Paris, Michel Lévy, 1858.

9 Théodore Agrippa d'Aubigné, Sa vie à ses enfants, Paris, Nizet, 1986.

10 Christine de Suède, Apologies. Vie de la reine Christine faite par elle-même, Paris, Le Cerf, 1994; Stanislas Auguste [Poniatowski], Mémoires, Paris, Institut d'études slaves, 2012. 
pas dans les mémoires publiés, car les éditeurs ne les ont pas jugées dignes de "servir à l'histoire de France" "11. Parfois aussi, le titre donné par l'éditeur est trompeur : les mémoires de Chaptal (1756-1832), intitulés Mes souvenirs sur Napoléon débutent en fait à la naissance de l'auteur et consacrent une dizaine de pages à ses études, de sa prime enfance à sa classe de philosophie puis à ses études de médecine à Montpellier ${ }^{12}$. Au contraire de leur prédécesseur Guizot, les ministres Victor Duruy (1811-1894) ou Jules Simon (1814-1896) nous livrent des souvenirs et commentaires très circonstanciés sur leur scolarité $^{13}$. Nombre de mémoires finissent donc par entremêler la grande Histoire et l'histoire personnelle de l'auteur, incluant ses souvenirs d'enfant et d'élève, ce qu'illustrent exemplairement les Mémoires d'Outre-Tombe de Chateaubriand.

\section{Autobiographies}

L'autobiographie se distingue des mémoires en ce que, dans son principe même, elle débute, non avec l'entrée de l'auteur dans le monde, mais avec son entrée dans la vie. Elle consacre donc une place significative aux événements mémorables de l'enfance et de l'adolescence, incluant l'instruction domestique ou scolaire reçue par l'auteur. L'autobiographie, J.-J. Rousseau l'a enflammée plus qu'il ne l'a inventée. Des souvenirs d'élèves et d'études apparaissent déjà ponctuellement dans des œuvres à caractère autobiographique comme celles de Guibert de Nogent (c. 1055-c. 1125) ${ }^{14}$, ou de Christine de Pizan (1364-c. 1430) ${ }^{15}$. Mais ce n'est que dans l'Europe de la Renaissance que de tels souvenirs commencent à s'écrire avec une fréquence notable, par exemple chez l'Augsbourgeois

11 C'est le cas de Nicolas Goulas (1603-1683), dont l'édition du XIX siècle (1879-1882) omet les nombreuses pages consacrées à son enfance et à son instruction. Noémi Hepp les a restituées dans Mémoires et autres inédits de Nicolas Goulas, gentilhomme ordinaire de la chambre du duc d'Orléans, Paris, Champion, 1995.

12 Jean-Antoine Chaptal, Mes souvenirs sur Napoléon, Paris, 1893. Ils ont été publiés par son arrièrepetit-fils. On apprend notamment dans ces mémoires que, sans grand rapport avec Napoléon, "à peine instruit des premiers éléments de la lecture, je prenais un plaisir tout particulier à feuilleter un Aristote qui m'était tombé sous la main"; sur cette lecture, voir infra.

13 Victor Duruy, Notes et souvenirs, 1811-1894, Paris, Hachette, 1901, 2 vol.; Jules Simon, Premières années, Paris, Flammarion, 1901; "Le collège de Vannes en 1830", Revue illustrée de Bretagne et d'Anjou, 1886.

14 Guibert de Nogent, Autobiographie [De vita sua], Paris, Les Belles Lettres, 1981.

15 Christine de Pizan, Le livre de l'advision Cristine, Paris, Champion, 2001, Cf. p. 109-113 : "Dit Cristine comment elle se mit à l'étude"; "Le plaisir que Cristine prenait à l'étude". 
Matthäus Schwarz, né en $1497^{16}$, le Valaisan Thomas Platter, né en $1499^{17}$, le Florentin Benvenuto Cellini, né en $1500^{18}$, ou le Milanais Jérôme Cardan, né en $1501^{19}$. Dès lors, des souvenirs d'apprentissages figureront régulièrement dans les récits de vie.

Les souvenirs d'élèves revêtent en fait une importance et un sens fort différents d'un auteur à l'autre. Ils peuvent résulter d'une simple exigence de continuité narrative : entre la naissance et l'entrée dans le monde, il faut bien donner quelques informations sur les événements qui y ont pris place, parmi lesquels le parcours d'instruction. Ils peuvent aussi s'inscrire dans un projet de connaissance de soi, qui met le vécu de l'enfance au service d'une compréhension de ce que deviendra l'adulte. Mais de ce point de vue, les expériences d'élèves sont jugées souvent moins constitutives ou prédictives de la personnalité future du narrateur que d'autres vécus enfantins. Frédéric Le Play observera, en 1864, que «Les hommes d'un jugement éprouvé qui ont bien voulu faire devant moi une rétrospective de leur vie, ont pu rarement reporter à l'enseignement reçu dans les écoles l'acquisition d'une partie essentielle de leur savoir $»^{20}$.

Les souvenirs d'apprentissage peuvent aussi donner l'occasion à leurs auteurs de tirer quelques leçons du passé, que ce soit pour l'édification de leurs descendants, seuls destinataires de nombre de récits de vie, ou pour celle de la société et de la postérité. Dans le premier cas, l'accent sera mis plutôt sur le comportement de l'ancien élève et sur ses rapports, bons ou mauvais, au travail scolaire et à l'instruction; dans le second, sur le contexte d'enseignement et d'apprentissage, dans ses contenus et méthodes, qu'a expérimenté l'auteur dans son enfance. Enfin, les souvenirs d'anciens élèves ont tout simplement partie liée avec ceux de l'enfance elle-même, dont tant de narrateurs disent le plaisir qu'ils ont de la revivre par l'écriture, quand bien même ce plaisir consisterait à régler des comptes et à solder de mauvais souvenirs. Le plus généralement, plusieurs de ces raisons conjuguent leurs effets pour conduire les anciens élèves à écrire leurs souvenirs.

16 Matthäus Schwarz, Un banquier mis à nu [Le livre des costumes]. Autobiographie de M. Schwarz, bourgeois d'Augsbourg, prés. par Philippe Braunstein, Paris, Gallimard, 1992. On peut considérer cette autobiographie comme la première à retracer des souvenirs d'élève en "bande dessinée", avant Riad Sattouf (1978-...), L'Arabe du futur. Une jeunesse au Moyen-Orient (1978-1987), Paris, Allary Éditions, 2014-2018, 4 vol.

17 La vie de Thomas Platter, écrite par lui-même [Lebensbeschreibung], Genève, Imprimerie Fick, 1862.

18 La vie de Benvenuto Cellini, écrite par lui-même (1500-1571), Paris, Scala, 1986.

19 Jérôme Cardan, Ma vie, Paris, Belin, 1991.

20 Frédéric Le Play, La Réforme sociale, Tours, Mame, 5e éd., 1874, livre 5, $2^{\mathrm{e}}$ partie, chap. 47, p. 36. 
Quels que soient les objectifs, conscients ou non, implicites ou explicites, que visent leurs auteurs, les souvenirs d'anciens élèves se caractérisent par leur régime de vérité : les auteurs donnent les faits qu'ils rapportent pour vrais. Ils ne font que rarement allusion à d'éventuelles lacunes ou imprécisions de leur mémoire. Si les hommes se plaignent plus volontiers de leur mémoire que de leur jugement, ce n'est pas le cas des auteurs de souvenirs, qui insistent souvent sur son excellence, dont ils sont assez fiers, exemples à l'appui. "J'apprenais cent cinquante vers par cœur dans une heure", écrit l'une ${ }^{21}$; "J'apprenais cent vers dans une demi-heure. Quelle mémoire j’avais!», surenchérit un autre ${ }^{22}$. Même si ce type de mémoire n'est pas celui où l'on va chercher des souvenirs d'élève, les mémorialistes l'incluent dans le contrat de confiance qui les lie à leurs lecteurs. D’autres sont d'ailleurs plus explicites sur la qualité de leur mémoire à long terme : "Ma mémoire me fournit les choses comme s'il n'y avait que trois jours qu'elles fussent arrivées", prétend le sieur de Gourville (1625-1703) pour se justifier de commencer à écrire ses mémoires à l'âge de soixante-seize $a_{n}{ }^{23}$. On peut dire que lorsqu'il écrit ses souvenirs, généralement au soir de sa vie, l'ancien élève les a déjà mis en mémoire, et sans doute ressassés, depuis longtemps ${ }^{24}$.

L'adolescence se prête particulièrement à ce stockage de souvenirs plus anciens, par la conscience qu'y prend l'adolescent(e) qu'une première période de sa vie est déjà révolue et qu'il importe d'en garder des traces. C'est l'âge où des adolescents commencent à tenir un journal, dans lequel leurs expériences d'élèves peuvent tenir une place significative, ce journal étant lui-même susceptible d'alimenter les souvenirs écrits ultérieurement. "C'est dans l'année 1510 que j'ai commencé à décrire en tous points ce qui m’arrivait", écrit le tout

21 Mémoires de Frédérique Sophie Wilhelmine, margrave de Bayreuth depuis l'année 1706 jusqu'à 1742. Écrits de sa main, Paris, Mercure de France, 1967, p. 59.

22 Charles Joseph, prince de Ligne, Mémoires, lettres et pensées, Paris, François Bourin, 1994, p. 53.

23 Jean Hérault, sieur de Gourville, Mémoires, Paris, Mercure de France, 2004, p. 27. Il ne consacre que quelques lignes à ses apprentissages, ses mémoires se bornant à relater "tout ce qui lui est arrivé de plus singulier".

24 Le parlementaire André Lefèvre d'Ormesson (1577-1665) rédige ainsi avec une extrême précision un "Mémoire des leçons que j'ai apprises en ma jeunesse" dans les différentes classes qu'il a suivies et sous les différents régents qui l'ont instruit, aux collèges du Cardinal Lemoine puis de Navarre, entre 1586 et 1592. Il s'en explique par le fait que "Dieu m'a fait la grâce d'avoir retenu par cœur jusqu'à la fin de mes jours toutes les poésies et les vers que j'avais appris en ma jeunesse. Il est vrai aussi qu'étant de loisir, je les relisais quelquefois pour m'en rafraîchir la mémoire». Cité par Adolphe Chéruel, De l'administration de Louis XIV (1661-1672), d'après les mémoires inédits d'Olivier d'Ormesson, Paris, Joubert, 1850, p. 203-207. 
premier mémorialiste de notre corpus, M. Schwarz, né en février 1497 et alors âgé de treize ans et dix mois exactement, comme il le précise ensuite. Sur les années qui précèdent, il "rassemble ses souvenirs" et demande à son père de combler ses "lacunes»" ${ }^{25}$. Il peut ainsi évoquer par l'image son apprentissage de la lecture et de l'écriture à l'âge de cinq ans et quatre mois; sa mère étant décédée, cet apprentissage est assuré par un écrivain public d'Augsbourg; il représente ensuite sa fréquentation d'une école publique à treize ans et quatre mois, sa sortie de l'école six mois plus tard, pour entrer au service de son père, négociant, puis, après un apprentissage de la comptabilité dans plusieurs villes d'Italie, à celui du banquier Fugger.

Les auteurs de souvenirs peuvent aussi disposer des traces matérielles de leurs activités d'élèves - cahiers, copies, extraits, livres scolaires... - conservés par eux-mêmes ou par leurs parents, pour des raisons sentimentales, et sur lesquels ils pourront éventuellement s'appuyer pour écrire leurs souvenirs. C'est le cas du Suisse Théophile-Rémy Frêne, fils de pasteur. Né en juin 1727, il écrit, entre avril et juin 1741, un "Roole, pour et par moy Theophile Remy Fresne"; ce "rôle" contient ses souvenirs depuis le début de 1732, où une place notable est consacrée à ses lectures et à ses premiers travaux d'écriture, réalisés sous la direction de son père, qu'il a conservés. Il dresse en effet le 30 juillet 1741 un "Catalogue de mes Manuscript [...]. En tout 30 Manuscript", qui comprennent des textes de fables, de géographie, de dictées d'orthographe, de catéchisme, de vocabulaire allemand; la plupart sont précisément datés, entre le 23 mai 1738 et le 20 février 1741, hormis « un petit feuillet volant, qui est le plus ancien de mes ecrit apres l'abc". À partir de juin 1741, son journal informe régulièrement et précisément sur ses études, sa scolarité et ses maîtres ou professeurs, dans le Jura bernois puis à Bâle, qui le conduiront jusqu'au pastorat ${ }^{26}$.

On ne dispose pas d'autant de précisions sur ce que pouvaient écrire "les grandes demoiselles" [de quatorze ou quinze ans] de l'aristocratique couvent de l'Abbaye-aux-Bois à Paris, dans les années 1770, mais chez elles, si l'on en croit les souvenirs d'Hélène Massalska (1763-1815), arrivée orpheline de Pologne en 1771, "c'était la mode d'écrire toute la journée leurs mémoires". La future princesse de Ligne précise que, pour les imiter, elle-même et d'autres filles

25 Matthäus Schwarz, Un banquier mis à nu..., op. cit., p. 114.

26 Théophile-Rémy Frêne, Journal de ma vie, éd. par André Bandelier, Porrentruy, Société jurassienne d'émulation, 5 vol., 1993-1994. 
plus jeunes, âgées de dix ou douze ans, écrivaient aussi les leurs ${ }^{27}$; ses propres "griffonnages" ont donc sans doute nourri les souvenirs d'élève qu'elle écrira ultérieurement. C'est dans cet objectif déclaré que le fils d'une couturière parisienne, Xavier-Édouard Lejeune, né en 1845, est saisi "à l'époque de ma quinzième année, de la manie d'écrire tout ce que je me rappelais de mon enfance", et cela "pour moi seul et pour retrouver plus tard, si je deviens vieux, tous les souvenirs de mes jeunes années". Ces souvenirs, qu'il intitule "Les étapes de ma vie", incluent force informations sur sa scolarité dans une école enfantine privée (5-7 ans), une école des Frères de Laon (7-11 ans), une autre école des Frères de Paris (quelques mois) et enfin une école mutuelle (12-14 ans), qu'il quitte pour "apprendre le dur métier de la vie" dans le commerce des tissus ${ }^{28}$.

Fils d'un comte italien et de la princesse Charlotte Bonaparte, JosephNapoléon Primoli donne un ultime exemple de stockage précoce de souvenirs d'élève, qui fait écho à celui de Schwarz, antérieur de trois siècles et demi. Né en 1851 à Rome, mais habitant Paris depuis 1853, il rédige en français "Mes souvenirs, écrits en 1861": ils entremêlent ses souvenirs personnels et des informations qui lui ont été rapportées. Il y mentionne ses apprentissages : depuis l'âge de deux ans, c'est sa mère qui lui a appris "à lire, à écrire, et tout ce que je sais"; à dix ans, son père lui donne des leçons de latin. En 1861, il décide de commencer à écrire "jour par jour, les différentes choses qui m’arriveront". Ces choses incluent sa scolarité au collège Rollin, où il entre en classe de $7^{\mathrm{e}}$ en octobre 1861 , et sur laquelle il se montre très prolixe ${ }^{29}$. Ces exemples de rédaction précoce de souvenirs d'élèves sont sans doute rares, mais il est clair que bien d'autres les consignaient "si ce n'est par écrit, au moins en idées", comme le soutient un diariste ${ }^{30}$. Ce dont Louis Aragon témoigne pour sa part, en citant Racine : "Ce que je sais le mieux, c'est mon commencement "31.

27 Apolline Hélène Massalska, Mémoires d'une écolière à l'Abbaye-aux-Bois à Paris (1771-1779), Oronle-Châtel, Association pour la conservation du château d'Oron, 2014.

28 Xavier-Édouard Lejeune, Calicot, Enquête de Michel et Philippe Lejeune, Paris, Montalba, 1984. Ces souvenirs ont été écrits entre 1860 et 1868, puis réécrits après 1891.

29 Giuseppe Primoli, Mémoires, 1851-1871, éd. par Massimo Colesanti et Valeria Petitto, Rome, Edizioni di Storia e Letteratura, 2012, p.3-21.

30 Cité dans Pierre Caspard, "Éducation et progrès. Ce que disent les écrits personnels", in Écrits au quotidien (XVIII ${ }^{e}$ - début XIX ${ }^{e}$ siècle), numéro spécial du Musée neuchâtelois, octobre 1996, p. 273-289.

31 Aragon, Je n'ai jamais appris à écrire ou les incipit, Paris, Skira, 1969. Aragon cite Petit Jean dans Les Plaideurs. 


\section{Témoignages}

Le souci de vérité, voire d'exactitude, peut notamment apparaitre chez ceux des anciens élèves qui veulent mettre le témoignage de leur expérience passée au service d'une connaissance des établissements scolaires qu'ils ont fréquentés, ou, plus généralement, du contexte éducatif qu'ils ont connu dans leur enfance et leur jeunesse, lorsqu'ils ont des raisons de penser que leurs lecteurs ont ou auront perdu le souvenir de ce contexte éducatif, général, régional ou local, révolu au moment où ils écrivent. C'est cette intention explicitement mémorielle qui anime déjà Guillaume Wallon, marchand de Beauvais, lorsqu'il rédige son Mémoire sur les petites écoles de Port-Royal, où il avait été élevé32, ces écoles ayant été détruites quatre ans après qu'il les a eu fréquentées, en 1656. Plus tard, de tels témoignages seront nombreux chez ceux qui auront effectué leur scolarité dans les décennies précédant les réformes de la Révolution et de l'Empire, puis celles du Second Empire et de la III ${ }^{e}$ République. Ils touchent à la fois au genre des mémoires, par leur souci d'informer sur des contextes "tels qu'ils ont réellement existé" et à l'autobiographie, par le nombre de souvenirs proprement personnels qu'ils contiennent. Au carrefour du souvenir d'élève et de l'histoire vécue des institutions scolaires, on peut y trouver de l'attendrissement sur soi comme du militantisme pédagogique, professionnel, religieux ou politique.

La plupart de ces témoignages ne constituent qu'un épisode, plus ou moins développé, dans le récit de toute une vie. Mais chez les mémorialistes qui écrivent au XIX ${ }^{\mathrm{e}}$ siècle, ils peuvent porter spécifiquement sur un établissement d'enseignement qu'ils ont jadis fréquenté : l'Abbaye-aux-Bois dans les années 1770 (Hélène Massalska), le collège de Vienne en 1788 (A. Yverain), la Maison de la Légion d'honneur d'Écouen en 1807-1815 (Thérèse-Mélanie Martin), le collège de Rouen en 1829-1835 (François Valentin Bouquet), le collège de Vannes en 1830 (Jules Simon), l'école primaire de S... vers 1850 (H. Barrau), la pension Beaujon à Paris sous le Second Empire (Brada), l'école normale de Nice en 1865 (Lucien Duc) et d'autres. La plupart des auteurs de ces monographies mémorielles, souvent rédigées à l'imparfait, appartiennent, assez logiquement, au monde

32 Cité dans Port-Royal. Anthologie établie, présentée et annotée par Laurence Plazenet, Paris, Flammarion, 2012, p. 248-252. 
enseignant $^{33}$. C'est que celui-ci a acquis au $\mathrm{XIX}^{\mathrm{e}}$ siècle une visibilité inédite : instituteurs, professeurs, maîtres ou maîtresses de pensions représentaient moins de $1 \%$ des auteurs de souvenirs d'élèves nés entre 1700 et 1749, mais $10 \%$ de ceux qui sont nés entre 1800 et 1851 (cf. infra). L'évolution du genre des souvenirs d'élèves dans le long terme a rapport avec l'identité sociale de leurs auteurs, qu'il convient donc de préciser.

\section{Les souvenirs d'élèves dans la suite des générations}

Les souvenirs publiés par d'anciens élèves nés entre la Renaissance et le milieu du XIX ${ }^{\mathrm{e}}$ siècle n'ont pas manqué d'être sollicités par les historiens de l'éducation et de l'école mais, très généralement, au prix de segmentations temporelles, par siècles ou par unités de temps liées à l'histoire même de l'école. Il importe de réinscrire ces segments dans une longue durée qui ne préjuge d'aucun principe de périodisation, mais fasse apparaître des cycles et des rythmes appelant, par eux-mêmes, analyses et réflexions.

\section{Une conjoncture des souvenirs d'élèves}

Le nombre des souvenirs d'études et d'apprentissages conservés et publiés s'est fortement accru sur l'ensemble de la période, ce qui n'est pas surprenant.En première analyse, cette évolution tient à la croissance de la population francophone, en France et en Europe, et au développement de la culture écrite, tant du côté des scripteurs que des lecteurs. Elle tient aussi à ce qu'il est convenu d'appeler la "montée de l'individualisme", même si, par-delà une certaine forme d'évidence, ce terme est plus problématique qu'explicatif ${ }^{34}$. Pourtant, cette croissance est loin d'être linéaire. C'est qu'elle agrège plusieurs autres facteurs d'explication.

33 S'agissant des seuls instituteurs, cf. Philippe Lejeune, "Les instituteurs du XIX ${ }^{\mathrm{e}}$ siècle racontent leur vie", incluant un "Répertoire des autobiographies écrites en France au XIX ${ }^{\mathrm{e}}$ siècle. Vies d'instituteurs", Histoire de l'éducation, n²5, 1985, p.53-104. Le répertoire présente 21 autobiographies d'instituteurs nés entre 1764 et 1858. Il est complété par une liste de 25 autobiographies d'instituteurs nés entre 1844 et 1913, publiées après 1914.

34 Cf. Claudette Delhez-Sarlet, Maurizio Catani (dir.), Individualisme et autobiographie en Occident, Bruxelles, Institut de sociologie, 1983. Les périodes étudiées vont de la Grèce ancienne au monde contemporain. 


\begin{tabular}{c|c|c|c}
\hline décennie & Nombre de naissances & décennie & Nombre de naissances \\
\hline 1490 & 3 & 1670 & 6 \\
\hline 1500 & 2 & 1680 & 5 \\
\hline 1510 & 1 & 1690 & 6 \\
\hline 1520 & 2 & 1700 & 10 \\
\hline 1530 & 3 & 1710 & 14 \\
\hline 1540 & 2 & 1720 & 21 \\
\hline 1550 & 4 & 1730 & 25 \\
\hline 1560 & 2 & 1740 & 38 \\
\hline 1570 & 4 & 1750 & 42 \\
\hline 1580 & 4 & 1760 & 72 \\
\hline 1590 & 7 & 1770 & 53 \\
\hline 1600 & 6 & 1780 & 51 \\
\hline 1610 & 11 & 1790 & 55 \\
\hline 1620 & 13 & 1800 & 49 \\
\hline 1630 & 6 & 1810 & 31 \\
\hline 1640 & 5 & 1820 & 24 \\
\hline 1650 & 5 & 1830 & 25 \\
\hline 1660 & 5 & 1840 & \\
\hline Total & 619 & & \\
\hline
\end{tabular}

Tableau 1 : naissance des auteurs de souvenirs d'élèves

Source : corpus de souvenirs défini supra, note 4.

Apparus d'une façon significative à la Renaissance, les auteurs de souvenirs d'élèves voient leur nombre s'accroître somme toute modérément durant les deux siècles qui suivent : on compte 2 ou 3 naissances de futurs auteurs par décennie au début du XVI ${ }^{e}$ siècle, 5 ou 6 à la fin du XVII (cf. tableau 1). Le nombre très élevé des naissances de futurs auteurs dans les décennies 16101620 constitue une exception intéressante. Elles sont celles des acteurs de la Fronde (1648-1653), qui écriront leurs mémoires dans la deuxième moitié du siècle, y intégrant, comme par raccroc, des souvenirs d'enfance et d'études. Peut-être la popularité nouvelle des Confessions de Saint Augustin, traduites en français par Robert Arnauld d'Andilly en 1649, a-t-elle contribué à légitimer l'évocation de souvenirs d'enfance et de jeunesse. En tout cas, de telles évocations n'apparaissaient aucunement dans les mémoires des acteurs d'un précédent épisode troublé de l'histoire de France, les guerres de Religion (1562-1598). Il existe bien une très forte poussée des naissances de l'ensemble des futurs mémorialistes dans les décennies 1550-15605, mais celle-ci ne se traduit par

35 Selon le site "Les écrits du for privé" (cf. note 4), près de la moitié de tous les mémorialistes du $\mathrm{XVI}^{\mathrm{e}}$ siècle sont nés durant ces deux seules décennies. 
aucune prise en compte de leur enfance dans les mémoires qu'ils écriront au début du siècle suivant. On peut voir dans cette différence l'indice et la conséquence des progrès de l'intérêt pour l'enfance et son éducation - y compris celui que l'on porte à la sienne propre - entre le début et la fin du XVII ${ }^{e}$ siècle $^{36}$.

Le début du XVIII ${ }^{\mathrm{e}}$ siècle, et particulièrement la décennie 1710 , voit au contraire une soudaine montée du nombre des naissances de futurs mémorialistes, y compris d'auteurs de souvenirs d'élèves. Armand de Pontmartin l'attribuait à la "puérile et ridicule prétention qui saisit les écrivains du XVIII" siècle de parler continuellement d'eux-mêmes, et d'entretenir le public de tout ce qu'ils avaient fait depuis le berceau" au motif que "ce qu'il y avait désormais de plus important dans la société, c'était un philosophe et un académicien ${ }^{37}$. Mais les "gens de lettres" qu'il vise sont loin d'être les seuls à parler de leur enfance et de leurs études. Conscients de vivre ou d'avoir connu un siècle de progrès économiques, culturels et intellectuels, de mobilité voire de bouleversements sociaux ${ }^{38}$, d'autres hommes et femmes du siècle ressentiront l'envie d'évoquer les changements qu'ils ont vécus ou dont ils ont été les témoins, et il se trouvera des lecteurs, donc des éditeurs, de plus en plus nombreux à s'intéresser à leurs écrits. D'autre part, la conscience des progrès passés et présents étayant l'ambition de modeler l'avenir, la place prise par les questions d'instruction publique et d'éducation nationale dans les débats du siècle, particulièrement dans sa seconde moitié, constituera une incitation particulière à inclure dans les souvenirs ceux des études et des apprentissages. Né en 1712, J.-J. Rousseau illustre bien la génération d'auteurs de souvenirs qui surgit durant cette décennie.

Né en 1768, Chateaubriand illustre, lui, une autre génération de mémorialistes, ceux qui naissent dans les décennies 1750 à 1770. Ces auteurs seront dans la force de l'âge durant les tourmentes révolutionnaires et impériales, qu'ils en aient été protagonistes - même modestes -, témoins ou victimes. Ils les évoqueront dans des mémoires et souvenirs qui paraîtront en masse sous la Restauration et la monarchie de Juillet. Beaucoup s'en tiendront aux

36 Cette observation peut nourrir le débat sur l'évolution du sentiment de l'enfance lancé jadis par Philippe Ariès dans L'enfant et la vie familiale sous l'Ancien Régime, Paris, Le Seuil, 1973.

37 Armand de Pontmartin, Mes mémoires. Enfance et jeunesse, Paris, 1882, p. 1.

38 Dès 1721, Montesquieu fait constater à son Persan qu'à Paris, "des révolutions enlèvent le pauvre, avec des ailes rapides, au comble des richesses". Rousseau fera un constat analogue, l'optimisme en moins : le mouvement social éloigne l'homme de l'état de nature. 
événements politiques ou militaires dans lesquels eux-mêmes et leur famille auront été mêlés. D’autres intégreront la dimension autobiographique léguée des générations antérieures en entremêlant la grande Histoire et leur histoire particulière, et donc leurs souvenirs de scolarité et d'apprentissages, ce dont témoignent justement les Mémoires d'Outre-Tombe.

Les décennies 1780-1790 voient une sensible baisse du nombre des naissances de futurs mémorialistes. Ils sont nés trop tard pour jouer un rôle actif dans les événements révolutionnaires et impériaux. En revanche, le chaos de leur parcours d'instruction à l'époque révolutionnaire donne à leurs souvenirs d'élèves un intérêt tout particulier.

Le nombre des naissances d'auteurs de souvenirs d'élèves semble continuer à fléchir dans la première moitié du XIX ${ }^{\mathrm{e}}$ siècle, mais cette baisse ne reflète que problématiquement l'importance de l'ensemble de la population publiant des souvenirs. D’un côté, après la génération de Chateaubriand, les mémorialistes, au sens ancien du terme, deviennent moins nombreux. "On a beau dire et beau faire, se désole Alphonse Allais à la fin du siècle, il y aura de moins en moins de gens qui auront connu Napoléon". D’un autre côté, le genre semble "s'être figé sous une forme sclérosée", le récit des "vies mémorables et majuscules" s'est effacé au profit des "récits de vies personnelles ou minuscules " ${ }^{39}$, qui se multiplient. Même si des souvenirs d'élèves majuscules et minuscules ont coexisté avant aussi bien qu'après ce demi-siècle, il est de fait qu'une grande vulgarisation du genre conduit à voir paraître des souvenirs de courtisanes, de trapézistes de cirque, de marchandes de modes, de maris et de femmes trompées, d'aliéné(e)s, de criminel(le)s, jusqu'à Cadichon qui publie ses Mémoires d'un âne (1860) et Erik Satie les Mémoires d'un amnésique (1912). La pertinence ou l'intérêt d'une évocation de souvenirs d'élève dans des mémoires de ce genre est minimal.

Surtout, les décennies qui vont de Napoléon ${ }^{\text {er }}$ à Guizot, Duruy et Ferry voient la mise en place d'un véritable système scolaire. Les programmes d'enseignement deviennent nationaux, les profils professionnels, sinon humains, des enseignants tendent à se standardiser, les cursus et trajectoires scolaires sont balisés et plus ou moins prévisibles, les diplômes que délivre l'école sont requis pour un nombre croissant d'emplois, masculins et, de plus en plus aussi,

39 Jean-Louis Jeannelle, Écrire ses mémoires au XX ${ }^{\mathrm{e}}$ siècle. Déclin et renouveau, Paris, Gallimard, 2008, p. 10. 
féminins, l'articulation entre instruction domestique et publique se distend. Dans l'ancien régime scolaire, les souvenirs d'anciens élèves informaient tout à la fois sur le quoi, le pourquoi, le qui, le quand et le comment des études et apprentissages. Le système scolaire une fois organisé nationalement, et même s'il subsiste des établissements confessionnels ou privés, ces souvenirs ne peuvent plus guère porter que sur les manifestations de la sociabilité scolaire, sur des figures d'enseignants ou de camarades remarquables, sur le rapport subjectivement vécu avec la discipline subie et l'enseignement dispensé ${ }^{40}$. Les spécificités proprement locales ou régionales qui caractérisaient le dispositif de l'ancien régime scolaire sont loin de disparaitre, mais s'estompent. On peut trouver en revanche dans les souvenirs le degré d'adhésion ou de distance avec ce qui est devenu une idéologie scolaire ${ }^{41}$. Sur un tout autre plan, les souvenirs d'élèves de grands auteurs peuvent apporter un éclairage à la singularité de destins exceptionnels; mais ceux d'A. Gide (né en 1869), K.-G. Jung (1875), L. Aragon (1897), J. Green (1900), N. Sarraute (1900), J.-P. Sartre (1905), S. de Beauvoir (1908) ou R. Gary (1914) appellent des approches essentiellement

40 Outre les textes officiels qui réglementent et régissent leur vie quotidienne, l'ouvrage de François Grèzes-Rueff et Jean Leduc, Histoire des élèves en France. De l'Ancien Régime à nos jours, Paris, Armand Colin, 2007, s'appuie sur les souvenirs de 75 anciens élèves : 8 d'entre eux sont nés avant 1800, 16 de 1801 à 1850, 19 de 1851 à 1900, 32 de 1901 à 1950. Les auteurs précisent bien qu'ils donnent au mot élève le sens contemporain de "tout individu scolarisé dans un établissement d'enseignement non universitaire", alors que dans le sens plus ancien du terme, que nous avons pour notre part retenu, il désigne plus largement "tout enfant recevant les leçons d'un maître" (p. 6). Ils excluent également les souvenirs d'élèves de l'enseignement professionnel. Mais les souvenirs d'écoliers doivent être distingués de ceux des collégiens ou lycéens, autant que l'enfance diffère de l'adolescence. S'agissant des premiers, Denis de Rougemont persifle : "Il existe des gens qui s'attendrissent sur leurs souvenirs de classe. C'est qu'ils les confondent avec ceux de leur enfance et les font indûment participer de la même grâce"; cf. "Les méfaits de l'instruction publique" (1929), in Trois pamphlets pédagogiques, Lausanne, L'Âge d'Homme, 1984, p. 123. Les Mémoires d'un écolier (1886) de Lucien Duc, né en 1849, illustrent remarquablement ce propos : "Les naïves aventures que j'ai racontées ont reporté mes lecteurs au temps heureux où ils étaient, eux aussi, écoliers; et, en me lisant, c'est à leurs propres souvenirs qu'ils ont souri. [...] Il est doux d'être ému ainsi”. C'est la raison que donne L. Duc à la poursuite de ses souvenirs dans les Souvenirs d'école normale (1891). S'agissant de l'enseignement secondaire, au contraire, Étienne Souriau croit voir prédominer "rancune" et "ressentiment" dans les souvenirs d'anciens élèves, mais il ne se fonde sur aucune étude empirique; cf. "Le souvenir de l'enfance", Journal de psychologie normale et pathologique, 1962, p. 15-57. Les souvenirs d'anciens élèves de collèges, de lycées, d'écoles primaires supérieures ou d'écoles normales sont en réalité divers et contrastés.

41 Gérard Poulouin, "Récits d'enfance, récits républicains", in Anne Chevalier, Carole Dornier (dir.), Le récit d'enfance et ses modèles, Caen, Presses universitaires de Caen, 2003, p. 63-89; Marie Françoise Chanfrault-Duchet, "La doxa scolaire dans les récits de vie", in Récits de vie, modèles et écarts, numéro spécial des Cahiers de sémiotique textuelle, no4, 1985, p.79-93. À partir d'une analyse de trois souvenirs d'élèves nés entre 1873 et 1899, elle juge que "la doxa scolaire tend à structurer le récit de vie, à lui donner une pertinence, un sens". 
littéraires ou philosophiques, en lien avec l'œuvre de leurs auteurs ${ }^{42}$ : écrits par des intellectuels célèbres, ils peuvent aussi faire figure de préquelles pour ceux qui connaissent les épisodes suivants, mais ne disent rien ou pas grand chose sur les contenus et les parcours d'éducation et d'apprentissage, qu'on ne connaisse déjà par d'autres sources.

En même temps, la banalisation des expériences d'élèves les fait entrer dans la mémoire collective. Cette collectivisation des souvenirs scolaires leur ouvre un champ spécifique dans le genre romanesque. Il est remarquable que le crépuscule de l'ancien régime scolaire, qui s'annonce dans les décennies 18201840, soit aussi le moment de la naissance des premiers auteurs d'un genre ou sous-genre nouveau, le roman d'enfant, qui consacre une place notable à sa vie scolaire, au collège ou au lycée surtout, dans un premier temps, puis aussi à l'école primaire ${ }^{43}$. En témoignent les noms de Ferdinand Fabre (né en 1827), Hippolyte Taine (1828), Jules Girardin (1832), Jules Vallès (1832), Alphonse Daudet (1840), Anatole France (1844), Octave Mirbeau (1848), Jean Aicard (1848), Gyp (1849) ou Pierre Loti (1850), en attendant Léon Frapié, Jules Renard, Colette, Alain-Fournier, Marcel Pagnol et bien d'autres. Mais quand bien même la plupart de ces romans possèdent une dimension autobiographique plus ou moins avérée, les souvenirs qui ont contribué à construire leurs personnages d'élèves requièrent une approche toute différente de celle des textes dont les auteurs ont passé un "pacte de vérité" avec leurs lecteurs ${ }^{44}$.

42 Par exemple, Philippe Lejeune a bien montré comment la dissemblance des récits que fait J.-P. Sartre de son entrée en lecture, en écriture et en littérature s'explique par le parcours intellectuel qui est le sien entre les moments où il écrit les Carnets de la Drôle de Guerre (1939-1940) et Les Mots (1963), P. Lejeune, Les brouillons de soi, Paris, Le Seuil, 1998, p. 165-253.

43 Aimé Dupuy, Un personnage nouveau du roman français. L'enfant, Paris, Hachette, 1933. Le chapitre VII, p. 229-278, est consacré à "L'enfant à l'école"; les dates de publication des 169 romans analysés s'échelonnent de 1876 à 1926, dont 85 antérieurement à 1914; Guillemette Tison, Une mosaïque d'enfants. L'enfant et l'adolescent dans le roman français (1876-1890), Arras, Artois Presses Université, 1998; Id. , Le roman de l'école au XIX siècle, Paris, Belin, 2004.

44 Analyser distinctement le "témoignage" des œuvres à caractère autobiographique et romanesque est une exigence méthodologique élémentaire, mais ne va pas de soi, en raison du pouvoir de séduction des seconds. Outre les souvenirs d'élèves, l'Histoire des élèves en France, op. cit., s'appuie ainsi sur une cinquantaine de récits romanesques; 11 de leurs auteurs sont nés entre 1799 et 1850, 23 entre 1851 et 1900 et 19 entre 1901 et 1950. L'article "Récits d'enfance, récits républicains...., cité note 41 , se fonde sur une vingtaine de récits publiés dans la deuxième moitié du XXe siècle, certains romancés, d'autres non. Dans Les écrivains français racontent l'école. 100 textes essentiels, Paris, Delagrave, 2001, Claude Thélot réunit des textes d'écrivains qui ressortissent aussi bien à leurs propres souvenirs d'élèves qu'à la fiction littéraire et aux réflexions sur l'école; une trentaine d'entre eux sont nés avant 1850. Sur le "pacte autobiographique" et les débats qu'il a suscités chez les littéraires, cf. Carole Allamand, Le "Pacte" de Philippe Lejeune ou l'autobiographie en théorie, Paris, Champion, 2018. 


\section{Du contexte des études à celui de leurs souvenirs}

Le milieu social où naît le futur auteur de souvenirs d'élève définit le contexte de ses apprentissages, les ressources humaines et financières disponibles, les valeurs et principes éducatifs de la famille et de la parentèle, les objectifs donnés à son instruction, que l'enfant les partage ou non, l'existence d'éventuels écarts n'étant pas le moindre intérêt de ces souvenirs, en ce qu'ils témoignent de la marge d'autonomie dont peuvent jouir les enfants et, éventuellement, des limites inférieures ou supérieures de leurs aptitudes et capacités. Ces milieux sociaux peuvent se caractériser de diverses façons mais, en première approximation, on se bornera à suivre leur évolution dans le temps selon une division tripartite commode : milieux de la noblesse, fortunée ou non, d'épée ou de robe, ancienne ou récente (exception faite de la noblesse d'Empire); peuple travaillant de ses mains, dans l'agriculture, l'artisanat, la domesticité...; entre les deux, et non sans tuilages avec les deux précédents, milieux de la bourgeoisie, grande, moyenne ou petite, commerçante, industrielle, rentière ou à talents.

\begin{tabular}{|c|c|c|c|c|c|c|c|c|c|c|}
\hline \multicolumn{2}{|l|}{$\begin{array}{l}\text { Années de } \\
\text { naissance }\end{array}$} & \multicolumn{3}{|c|}{ Hommes } & \multirow[t]{2}{*}{ Total } & \multicolumn{3}{|c|}{ Femmes } & \multirow[t]{2}{*}{ Total } & \multirow{2}{*}{$\begin{array}{l}\text { Total } \\
\text { général }\end{array}$} \\
\hline & & $\begin{array}{l}0 \\
0 \\
0 \\
0 \\
0 \\
0 \\
0 \\
z\end{array}$ & 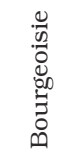 & $\begin{array}{l}\stackrel{0}{\vec{Z}} \\
\text { D. }\end{array}$ & & $\begin{array}{l}0 \\
0 \\
0 \\
0 \\
0 \\
0 \\
z\end{array}$ & $\begin{array}{l}0 \\
\bar{n} \\
0 \\
0 \\
0 \\
\vdots \\
0\end{array}$ & $\begin{array}{l}\vec{u} \\
\vec{\Xi} \\
\mathbb{0} \\
\end{array}$ & & \\
\hline \multirow[t]{2}{*}{ 1490-1699 } & $\mathrm{N}$ & 40 & 26 & 12 & 78 & 12 & 11 & 2 & 25 & 103 \\
\hline & $\%$ & 51 & 34 & 15 & 100 & 48 & 44 & 2 & 100 & \\
\hline \multirow[t]{2}{*}{$1700-1749$} & $\mathrm{~N}$ & 20 & 27 & 20 & 67 & 6 & 2 & 1 & 9 & 76 \\
\hline & $\%$ & 30 & 40 & 30 & 100 & 67 & 22 & 11 & 100 & \\
\hline \multirow[t]{2}{*}{ 1750-1799 } & $\mathrm{N}$ & 61 & 103 & 48 & 212 & 28 & 11 & 5 & 44 & 256 \\
\hline & $\%$ & 29 & 48 & 23 & 100 & 64 & 25 & 11 & 100 & \\
\hline \multirow[t]{2}{*}{$1800-1851$} & $\mathrm{~N}$ & 11 & 77 & 54 & 142 & 3 & 34 & 5 & 42 & 184 \\
\hline & $\%$ & 8 & 54 & 38 & 100 & 7 & 81 & 12 & 100 & \\
\hline N Total & & 132 & 233 & 134 & 499 & 49 & 58 & 13 & 120 & 619 \\
\hline
\end{tabular}

Tableau 2 : origine sociale des mémorialistes évoquant leurs souvenirs d'ancien(ne)s élèves (1490-1851)

Source : Mémoires et souvenirs du corpus défini supra, note 4.

La part relative des auteurs de souvenirs appartenant à chacun de ces milieux traduit des évolutions inégalement attendues. Le déclin de la contribution des nobles est massif et continu chez les hommes. Chez les femmes nobles nées 
dans la seconde moitié du XVIII siècle, cette contribution s'élève très fortement par rapport au siècle précédent, avant de s'effondrer au niveau de celle des hommes au siècle suivant; il faut voir dans cette évolution particulière le rôle joué par les femmes dans la transmission de la mémoire de leur famille, de leur milieu social, de leur ordre, au lendemain de la tourmente révolutionnaire ${ }^{45}$.

Corrélativement, les mémorialistes, hommes et femmes, d'origine bourgeoise voient logiquement leur nombre s'accroître avec le temps. Il en va de même pour les mémorialistes masculins d'origine populaire, alors que la proportion des femmes de même origine stagne tout au long de la période, à un niveau très faible. Au total, la part des femmes évoquant des souvenirs d'instruction est de $27 \%$ chez les mémorialistes nobles, $20 \%$ chez les bourgeois, $9 \%$ seulement dans les milieux populaires.

La profession ou la position du mémorialiste au moment où il écrit ses souvenirs ${ }^{46}$ conditionne, de son côté, à la fois la nature et l'objectif de la mise en récit de ses souvenirs d'élève, le jugement qu'il peut rétrospectivement porter sur son parcours d'apprentissage et la façon dont celui-ci a orienté - ou non son avenir social et professionnel.

La gamme des professions ou activités exercées par les mémorialistes masculins est très large (cf. tableau 3). Encore faut-il observer qu'il est fréquent que chacun d'eux en ait exercé plusieurs, voire de nombreuses, successivement ou simultanément : il y a même là un puissant motif à écrire ses souvenirs, le récit attestant la cohérence, voire la simple continuité des différents personnages que le mémorialiste a été et des différents rôles qu'il a joués dans sa vie ${ }^{47}$. Attribuer à un mémorialiste une activité unique ne constitue en tout cas qu'une approximation : il y a une part d'arbitraire à avoir classé Michelet

45 Henri Rossi, Mémoires aristocratiques féminins, 1789-1848, Paris, Champion, 1998.

46 On considère que le moment de rédaction des souvenirs se situe vers la fin de la vie active des auteurs, même si ce n'est pas toujours le cas; par ailleurs, la rédaction de souvenirs peut s'étendre sur de nombreuses années.

47 L'histoire sociale tangente ici ce qu'écrit Paul Ricœur sur l' "identité narrative», Temps et récit, Paris, Le Seuil, 3 vol., 1983-1985. Dans Soi-même comme un autre, Paris, Le Seuil, 1990, il évoque "le caractère évasif de la vie réelle, qui requiert le secours de la fiction pour organiser cette dernière rétrospectivement dans l'après-coup" et atteindre "l'unité narrative de la vie", p. 191. Georges Gusdorf voit pour sa part dans l'écriture autobiographique "la recherche du sens de sa destinée", la tentative de "définir le sens d'une vie». Il est vrai qu'il se flatte de ne s'intéresser qu'aux "œuvres maîtresses du genre", abandonnant les autres aux "sociologues de la première personne" et aux "professionnels de l'autobiographie de masse", les uns et les autres affligés du "postulat de la platitude universelle"; cf. Lignes de vie 1: Les écritures du moi, Paris, Odile Jacob, 1990, p. 24-25; et Lignes de vie 2 : Auto-bio-graphie, Paris, Odile Jacob, 1990. 


\begin{tabular}{|c|c|c|c|c|c|}
\hline \multirow[t]{2}{*}{ Profession ou activités } & \multicolumn{4}{|c|}{ Années de naissance } & \multirow[t]{2}{*}{ Total } \\
\hline & $\begin{array}{c}1490- \\
1699\end{array}$ & $\begin{array}{c}1700- \\
1749\end{array}$ & $\begin{array}{c}1750- \\
1799\end{array}$ & $\begin{array}{c}1800- \\
1851\end{array}$ & \\
\hline Écrivains, hommes de lettres & 12 & 17 & 25 & 31 & 85 \\
\hline Officiers supérieurs & 23 & 10 & 42 & 6 & 81 \\
\hline Politiques & 3 & 5 & 30 & 11 & 49 \\
\hline Haute administration & 3 & 1 & 16 & 3 & 23 \\
\hline Magistrats, avocats & 5 & 4 & 10 & 3 & 22 \\
\hline Enseignants, pédagogues & 1 & 1 & 11 & 16 & 29 \\
\hline Hommes de science & 1 & 3 & 7 & 11 & 22 \\
\hline Historiens, érudits & 4 & 3 & 7 & 6 & 20 \\
\hline Haut clergé & 4 & 1 & 1 & 1 & 7 \\
\hline Bas clergé & 4 & 5 & 3 & 4 & 16 \\
\hline Pasteurs & 4 & 2 & 1 & 4 & 11 \\
\hline Artistes & 1 & 2 & 4 & 4 & 11 \\
\hline Comédiens & 0 & 3 & 6 & 4 & 13 \\
\hline Soldats, sous-officiers & 0 & 0 & 14 & 1 & 15 \\
\hline Industriels, négociants & 4 & 3 & 8 & 4 & 19 \\
\hline Artisans, ouvriers & 3 & 5 & 9 & 16 & 33 \\
\hline Paysans & 0 & 0 & 0 & 0 & 0 \\
\hline Divers & 6 & 2 & 17 & 18 & 43 \\
\hline Total & 78 & 67 & 211 & 143 & 439 \\
\hline
\end{tabular}

Tableau 3 : profession ou activités des auteurs de souvenirs d'élèves (hommes)

\begin{tabular}{l|c|c|c|c|c}
\hline \multirow{2}{*}{ Profession ou activités } & \multicolumn{3}{|c|}{ Années de naissance } & \multirow{2}{*}{ Total } \\
\cline { 2 - 5 } & $\begin{array}{c}\mathbf{1 4 9 0} \\
\mathbf{1 6 9 9}\end{array}$ & $\begin{array}{c}\mathbf{1 7 0 0} \\
\mathbf{1 7 4 9}\end{array}$ & $\begin{array}{c}\mathbf{1 7 5 0}- \\
\mathbf{1 7 9 9}\end{array}$ & $\begin{array}{c}\mathbf{1 8 0 0 -} \\
\mathbf{1 8 5 1}\end{array}$ & \multicolumn{2}{c}{46} \\
\hline $\begin{array}{l}\text { Mémorialistes } \\
\text { (d'elles-mêmes, de leur famille) }\end{array}$ & 11 & 3 & 24 & 8 & 30 \\
\hline Femmes de lettres & 5 & 3 & 3 & 19 & 17 \\
\hline Religieuses & 7 & 1 & 5 & 4 & 4 \\
\hline Artistes & 0 & 0 & 1 & 3 & 8 \\
\hline Comédiennes & 0 & 1 & 4 & 3 & 7 \\
\hline Enseignantes, pédagogues & 0 & 1 & 4 & 2 & 8 \\
\hline Divers & 2 & 0 & 4 & 2 & $\mathbf{1 2 0}$ \\
\hline Total & $\mathbf{2 5}$ & $\mathbf{9}$ & $\mathbf{4 5}$ & $\mathbf{4 1}$ & $\mathbf{2}$ \\
\hline
\end{tabular}

Tableau 4 : profession ou activités des auteurs de souvenirs d'élèves (femmes)

Source : Mémoires et souvenirs du corpus défini supra, note 4. 
parmi les historiens, comme nous l'avons fait ici, plutôt que parmi les professeurs, voire les écrivains, et la remarque vaudrait pour bien d'autres. Il serait également important, mais difficilement formalisable, de tenir compte de l'ordre de succession des professions ou activités que le mémorialiste a effectivement exercées, entre la fin de sa formation et, donc, de son expérience d'élève, et sa position sociale au moment où il écrit ses souvenirs. Ceci reviendrait à interroger la logique même d'une carrière ou d'un parcours de vie, entre l'investissement initial de formation, qui a pu donner - ou non - du sens à l'expérience d'élève, et ce qui revient ensuite aux dispositions, au talent, aux relations, au hasard et aux circonstances.

À ces importantes réserves près, deux types d'activités se détachent nettement chez les hommes. La première est celle d'écrivain ou d'homme de lettres, au sens large, de romancier ou poète à journaliste. Nous avons rangé dans cette catégorie les auteurs de souvenirs qui doivent à cette activité l'essentiel de leur notoriété, même s'ils en ont par ailleurs exercé d'autres; ils s'y trouvent donc sur-représentés. La rédaction de souvenirs s'inscrit pour eux dans la continuité directe d'une activité habituelle d'écriture. La qualité du récit en bénéficie très largement, mais la frontière avec la fiction peut s'en trouver transgressée. De tous les souvenirs d'anciens élèves, la lecture de ceux des écrivains demande sans doute la plus grande circonspection, dans une perspective spécifiquement historienne ${ }^{48}$.

Sur l'ensemble de la période, la seconde position sociale la plus notable des mémorialistes est celle d'officier supérieur. Les maréchaux, généraux, colonels..., écrivent leurs souvenirs au lendemain des guerres auxquelles ils ont participé. D'où leur forte sur-représentation après celles de la Révolution et de l'Empire, encore plus marquée pour les sous-officiers et simples soldats, totalement silencieux en dehors de ce contexte historique exceptionnel. Pour des raisons voisines, les fonctions politiques, administratives et judiciaires ont également suscité chez ceux qui les ont exercées un surcroît d'écritures mémorielles au lendemain de la chute de l'Empire.

Moins liée au temps court, l'évolution de la plupart des autres professions exercées par les mémorialistes est plus ou moins conforme à ce qu'on peut attendre de celle de la société française - et, plus largement, occidentale - en

48 Goethe intitule justement ses mémoires Fiction et vérité [Aus meinem Leben. Dichtung und Wahrheit] (1833); Aragon évoque son enfance sous le titre Le mentir-vrai (1964). A. de Pontmartin juge que, pour la véracité d’un récit, "l'obscurité même du narrateur est un avantage», Mes mémoires, op. cit., p.3. 
général. Ainsi, la représentation des enseignants s'est très fortement accrue, comme il a déjà été souligné. À peine moins marquée est la progression des hommes de science. Pour les uns comme pour les autres, les souvenirs d'anciens élèves présentent l'intérêt de se situer dans une continuité particulière avec leur profession future. En comparaison, la présence des prêtres et des pasteurs n'évolue guère. On observe une forte sur-représentation de ces derniers par rapport aux membres du clergé catholique, compte tenu de leur importance respective dans la population; elle tient à deux raisons : le souci d'une mémoire familiale, qui ne concerne pas les prêtres, et celui de témoigner sur la situation difficile faite à une minorité religieuse, y compris au plan éducatif, entre les guerres de Religion et le début du XIX ${ }^{\mathrm{e}}$ siècle. Artistes et comédiens, d'une part, industriels et négociants, d'autre part, sont significativement représentés chez les auteurs de souvenirs; les mémoires des premiers répondent à une curiosité publique, les seconds veulent plutôt témoigner des raisons du succès ou, plus rarement, de l'échec de leurs entreprises. Par ailleurs, le nombre de mémorialistes ne s'inscrivant pas dans les profils les plus canoniques s'accroît avec le temps, ce qui traduit la vulgarisation du genre mémoriel; figurent ainsi dans la catégorie "divers" des médecins, des architectes, des libraires, des marins ou officiers de marine, des valets de chambre mais aussi des assassins, et quelques inconnus.

Remarquable, enfin, est le contraste entre les souvenirs d'élèves dans deux milieux populaires : les artisans et ouvriers d'une part, les paysans d'autre part. L'existence de mémorialistes parmi les premiers connaît une forte croissance, pour deux raisons au moins. L'une est la présence parmi eux d'une élite de plus en plus nombreuse à être instruite, parfois militante; leurs souvenirs d'anciens élèves plaident souvent pour les bienfaits de l'instruction, qu'ils s'estiment satisfaits ou non de celle qu'ils ont eux-mêmes reçue, et dont ils jugent donc utile d'évoquer le souvenir. Ainsi, François-Xavier Gressot (17831868), fils d'un brasseur et aubergiste de Grandvillars (Territoire de Belfort), destine ses mémoires à ses descendants et relate longuement, sans lésiner sur les jugements et commentaires, son parcours d'instruction scolaire et autodidactique à Delémont, Porrentruy et Mulhouse, puis la dizaine de métiers qu'il a exercés, entre travail manuel et commerce, pour finir sa vie dans une certaine aisance. Il crédite l'instruction qu'il a reçue ou qu'il s'est donnée de lui avoir permis de "profiter de tous les hasards qui ont pu se présenter dans le cours de sa vie" et en tire la leçon : "Je conseille donc à mes enfants et à 
mes neveux et petits-neveux de chercher à tout apprendre et à faire pour cela les plus grands sacrifices ${ }^{49}$.

L'exemple de F.-X. Gressot témoigne aussi, plus généralement, de la mobilité géographique, professionnelle, parfois sociale dont les ouvriers et artisans sont de plus en plus nombreux à pouvoir bénéficier, dans un contexte de développement du capitalisme et de l'urbanisation. Cette dernière raison, et celle-là seule, a son exact contraire dans l'explication de l'absence totale de paysans parmi les mémorialistes. En trois siècles et demi, durant lesquels ils ont pu représenter au moins les deux-tiers de la population française, aucun paysan, même fortuné et instruit, n'a jamais écrit de mémoires ${ }^{50}$. Ce n'est pas parce que, en tant que paysans, "ils n'avaient pas accès à la culture écrite " ${ }^{51}$, mais en raison de leur enracinement. "Naître, vivre et mourir dans la même maison " peut être un idéal (selon Sainte-Beuve), mais celui qui le vit n'a rien à raconter qui ne soit déjà inscrit, d'une façon ou d'une autre, dans la terre, la pierre, les archives de sa paroisse ou de sa commune, dans quelques livres de raison ou papiers de famille, ou dans la mémoire collective du lieu ${ }^{52}$. C'est en ce sens proprement social que l'individualisme, comme antithèse à l'enracinement collectif, peut conduire à la rédaction de mémoires.

À la différence des hommes, les femmes qui évoquent leurs souvenirs d'études et d'apprentissages n'ont que très minoritairement exercé une profession identifiable, ce qui n'est nullement synonyme d'absence de vie sociale. Sous l'Ancien Régime, la majorité d'entre elles, d'origine noble, se signalent princi-

49 Histoire de ma vie. Au cour de l'industrialisation alsacienne et jurassienne. François-Xavier Gressot: artisan, contremaître et négociant (1783-1868), Alain Cortat (éd.), Neuchâtel, Alphil, 2002. Avant lui encore, et en des termes tout à fait analogues, le tisserand sarthois Louis Simon, né en 1741, énonce dans ses mémoires un principe qu'il a fait sien: "Faut toujours apprendre quand on est à même, parce que l'on sait bien où l'on vive (sic) mais on ne sait point où l'on meurt". Anne Fillon, Louis Simon, villageois de l'ancienne France, Rennes, Éditions Ouest-France, 1996, p. 41.

50 Trois ou quatre mémorialistes ont fini leurs jours comme "cultivateurs ", mais ils avaient précédemment ou simultanément exercé d'autres activités.

51 "Il ne peut y avoir d'écriture paysanne - mémoires, témoignages - que de la part de ceux qui ont cessé d'être paysans, par le fait même de leur accession à la culture écrite", écrit Jean-Marie Goulemot, dans son introduction aux Mémoires de Valentin Jamerey-Duval. Enfance et éducation d'un paysan au XVIIIe siècle, Paris, Le Sycomore, 1981, p. 17. J.-M. Goulemot met en cause les fins et la logique qui seraient celles du "dispositif culturel de l'Ancien Régime", mais il sous-estime la pratique des écritures ordinaires dans les campagnes, depuis la fin du Moyen Âge. D’ailleurs, V. Jamerey-Duval n'est pas paysan : son père est charron, donc artisan, son parâtre est journalier agricole, donc ouvrier, et lui-même n'a jamais été "homme de la glèbe" mais berger, occupation assez répandue chez les jeunes des deux sexes dans les campagnes, avant qu'ils en exercent d'autres.

52 Françoise Zonabend, La Mémoire longue. Temps et histoire au village, Paris, Presses universitaires de France, 1980. 
palement comme mémorialistes, d'elles-mêmes et de leur famille, mari, mère, tante, enfants... Le rôle des femmes comme gardiennes de la mémoire familiale n'est plus à souligner. Mais on observe ensuite un remarquable basculement, lié en partie à l'évolution de leurs origines sociales : nées après la Révolution, et désormais majoritairement d'origine bourgeoise, c'est davantage comme femmes de lettres, romancières, poétesses, qu'elles écrivent leurs mémoires.

Certaines d'entre elles ont pu jouer un rôle politique non négligeable, au moins dans la société aristocratique et princière qu'abolira la Révolution, mais les positions permettant l'exercice statutaire d'un pouvoir militaire, administratif, judiciaire ou autre leur ont toujours été fermées et le sont restées au début du siècle suivant. Ce sont ces perspectives qui déterminent, en amont, l'instruction reçue par les filles dans l'ancien régime scolaire, et non l'inverse. Leurs souvenirs d'éducation et d'instruction présentent donc la particularité d'être quasi totalement dénués de toute considération, explicite ou implicite, sur des perspectives ou finalités professionnelles. Ils touchent essentiellement à leur formation générale, littéraire, historique, artistique. Comme chez les hommes, l'instruction religieuse tient le plus souvent une place minime dans leurs souvenirs, excepté pour ce qui concerne la préparation à leur première communion $^{53}$. Les femmes sont encore moins disertes sur leur apprentissage des travaux d'aiguille, sur lesquels elles nous laissent cependant quelques jugements contrastés ${ }^{54}$.

53 Exactement comme pour les garçons, l'instruction proprement catéchétique laisse souvent aux filles un souvenir critique, en raison des rapports problématiques entre simple mémorisation et vraie compréhension du dogme, ressentis aussi bien par elles-mêmes que par leurs catéchistes. Cf. Pierre Caspard, "Des écrits personnels à l'histoire : les gens ordinaires sont-ils importants?", in L'histoire contemporaine et les écrits en Suisse romande (XIX ${ }^{e}-X X^{e}$ siècles), numéro spécial de la Revue historique neuchâteloise, $\mathrm{n}^{\circ}$ 1-2, 2014, p. 15-30. L'idée que, dans l'instruction religieuse, les Églises, catholique ou protestantes, se seraient satisfaites d'une mémorisation sans compréhension est un mythe historiographique "républicain", qui semble inoxydable mais que contredisent formellement, entre autres, les souvenirs d'élèves. Les souvenirs d'éducation et d'instruction religieuses revêtent bien sûr une tonalité différente chez les femmes (comme chez les hommes) ayant vécu une expérience spirituelle ou mystique, pouvant se prolonger dans une vocation religieuse, comme c'est le cas pour une quinzaine d'entre elles. Cf. Louis Châtellier, Philippe Martin (dir.), L'écriture du croyant, Turnhout, Brepols, 2005; cf. aussi les extraits d'autobiographies publiés par Isabelle Poutrin, Le Voile et la plume. Autobiographie et sainteté féminine dans l'Espagne moderne, Madrid, Casa de Velasquez, 1995, p. 283-420.

54 Ainsi, Catherine de la Guette (1613-1676) écrit qu'elle a "toujours été d’une humeur plus portée à la guerre qu'aux exercices tranquilles de mettre les poules à couver et de filer la quenouille, quoique l'on dise qu'une femme ne doit savoir que cela"; et ses parents n'ont fait aucun obstacle à ce qu'elle apprenne l'escrime et le maniement des armes à feu. Cf. Mémoires de Madame de la Guette, écrits par elle-même, éd. par M. Moreau, Paris, Jannet, 1856, p.34. Évoquant le contenu de ses études, Christine de Suède (1626-1689) se flatte d'avoir appris "à se servir de toutes les armes passablement bien", affirmant même "avoir une aversion et une antipathie invincibles pour tout ce que font les 


\section{Parcours, apprentissages, acteurs : trois aperçus}

Les souvenirs d'anciens élèves constituent, d'une façon spécifique et non substituable, une source d'analyse et de compréhension des parcours d'apprentissage, de leurs contenus et modalités et du rôle joué par certains de leurs acteurs.

\section{Parcours d'apprentissage}

L'histoire de l'enseignement segmente ordinairement les lieux et institutions des apprentissages entre famille, précepteurs, écoles publiques ou privées, collèges, couvents, séminaires, lycées, etc. En réalité, de la Renaissance à la fin de l'ancien régime scolaire, les parcours sont presque toujours composites. En outre, ils se déroulent dans un contexte précis, familial, local, régional, éventuellement affecté par des événements contingents : décès familial, revers de fortune, guerre ou révolution. Les parcours individuels rapportés dans les souvenirs peuvent ainsi se comprendre en termes de choix et d'arbitrage entre un ensemble d'opportunités et de contraintes, analysées par la famille, les proches et les enfants eux-mêmes. Même si elles résultent nécessairement de divers principes de sélection, les informations données par le mémorialiste, largement factuelles, ne laissent qu'une place réduite aux reconstructions ultérieures. Il en va un peu différemment du ressenti ou du jugement qu'il porte par la suite sur ces apprentissages, en fonction de ce que lui inspirent le déroulement de son propre parcours et l'évolution générale de la société.

S'agissant de la réalité même des parcours d'instruction, les souvenirs d'anciens élèves respectent naturellement, dans ses plus grandes lignes, ce que nous savons par d'autres sources sur l'histoire de la scolarisation des petits Français des deux sexes. Ainsi, quasiment aucun enfant de noble n'a jamais été scolarisé dans une école communale ou de charité. Un cas sans doute exceptionnel est celui de la petite Henriette de Monbielle d'Hus, future marquise de Ferrières. Elle est envoyée dans son jeune âge "chez les religieuses [ursulines] qui montraient à lire aux filles du peuple; je me trouvais

femmes", Apologies...op. cit., p.21. Au contraire, Dorothée, future duchesse de Dino (1793-1862) déclare "être une bonne ouvrière dans les ouvrages de l'aiguille, et cela me plait". Souvenirs de la duchesse de Dino, Paris, 1901, chap. 3. De même, George Sand (1804-1876) regrette d'avoir été privée dans son enfance d'apprendre les travaux d'aiguille, par la faute de sa grand-mère, qui l'y jugeait impropre; elle ne se les est fait enseigner que sur le tard et s'y livre alors "avec passion", Histoire de ma vie, chapitre 9, "Travaux d'aiguille moralement utiles aux femmes". 
donc confondue avec la canaille de la ville [de Thouars] ${ }^{55}$ ". C'est un souci d'économie qui guide sa mère, peu instruite, surchargée de travail et, surtout, dépourvue de tout sens de la pédagogie : "le livre me volait à la tête et je ne pouvais rien apprendre". Finalement, "au contact de cette canaille, je devins un vrai polisson, mentant, parlant en mauvais termes. Il fallut m'en retirer". Inversement, les enfants appartenant aux milieux populaires les plus modestes n'ont jamais fréquenté, sauf rares exceptions, un collège ou un lycée, ni n'ont reçu de leçons d'un précepteur ou d'une gouvernante; tout au plus quelques enfants de domestiques ont-ils été admis à partager des leçons données aux enfants de leurs maîtres. Mais entre ces situations extrêmes, où l'on peut voir le poids des destins sociaux, les parcours composites abondent. Une analyse sérielle de l'ensemble des souvenirs permettrait d'établir des parcours-types au fil du temps. On n'en donnera ici qu'un exemple, qu'on se gardera bien de qualifier d'exemplaire, mais qui est significatif des expériences d'élèves pouvant se rencontrer dans l'ancien régime scolaire.

Ce parcours est celui d'un mémorialiste qui occupe une place moyenne dans le spectre social, Jean-Joseph de Verneilh-Puyraseau (1756-1839), né à Nexon (1 860 habitants en 1793), dans la Haute-Vienne. Il se déclare issu d'une famille "ancienne et honnête, mais peu fortunée ces derniers temps"; son père est notaire royal. Devenu avocat lui-même, il sera député sous différents régimes, entre 1791 et 1830, ses mémoires étant publiés en $1836^{56}$.

Il rapporte que c'est son père qui lui a "donné ses premières leçons de lecture». Il partage ensuite les leçons données aux deux enfants d'une famille voisine et amie de Nexon par un "instituteur" ${ }^{57}$, "d'un certain âge et d'une grande mansuétude». À l'âge de "huit ou neuf ans", il est envoyé à Saint-Yrieixla-Perche (6200 habitants en 1793), chez un oncle médecin, sans enfant.Il y fréquente l'"école solitaire" tenue par un "maitre" qui, outre lui-même, n'a qu'une poignée d'élèves, dont un unique pensionnaire. Il fréquente ensuite l'école tenue par le "maître en titre" de Saint-Yrieix, qui compte soixante élèves divisés en plusieurs "classes", que le maitre enseigne plus ou moins successivement; les élèves des autres "classes" en profitent pour tenter de s'échapper

55 Souvenirs en forme de mémoires de la Marquise de Ferrières, Bonnes, les Gorgones, 1998, p.3-4.

56 Jean-Joseph de Verneilh-Puyraseau, Mes souvenirs de soixante-quinze ans, Limoges, 1836.

57 Au XVIII ${ }^{\mathrm{e}}$ siècle, "instituteur" peut être synonyme de "précepteur". 
et s'ébattre au dehors. Il y apprend la grammaire latine en mémorisant, sans trop les comprendre, le "rudiment de Gaudin" 58 et "un certain Despautère" 59 .

Après examen de son niveau, il entre en 1770, à l'âge de quatorze ans, en classe de $3^{\mathrm{e}}$ (il espérait entrer en $2^{\mathrm{e}}$ ) au collège de Limoges ${ }^{60}$, où il poursuit ses études jusqu'en philosophie. De ses années de collège, il évoque le souvenir, plutôt favorable, des différents régents sous lesquels il a étudié, des prix et couronnes qu'il a remportés et des "jours de gloire scolastique" qu'ils lui ont valus, ainsi que d'une "philippique en vers" rédigée par un élève contre ses professeurs, "qui fit grand bruit". Durant ses classes de $3^{\mathrm{e}}$ et $2^{\mathrm{e}}$, il est précepteur du fils d'un parent qui l'a accueilli en pension; il doit donner "les premiers soins" à son "jeune élève", alors que son parent lui-même, ancien capitaine, "facilite son éducation". Il entre ensuite à la faculté de droit de Toulouse.

Ce parcours singulier ${ }^{61}$ résulte d'un contexte familial et local précis, mais révèle quelques traits plus généralement observables dans les souvenirs : un apprentissage de la lecture à domicile (ici, par le père; plus souvent, par la mère); la flexibilité de la fonction préceptorale, évoquée ici chez un homme d'âge mûr, qui enseigne les deux enfants d'une famille, avec adjonction d'un enfant d'une famille amie, puis chez un collégien, qui enseigne le plus jeune fils d'une famille où il est pensionnaire; la polysémie du terme "école", qui désigne aussi bien une "école solitaire" (expression peu usitée) fréquentée par une poignée d'enfants, qu'une école communale, qui en réunit plusieurs dizaines ${ }^{62}$.

Deux des épisodes de ce parcours se prêtent particulièrement à une confrontation systématique avec ce que disent les autres souvenirs d'élèves : l'apprentissage de la lecture et l'enseignement préceptoral. Nous nous bornerons ici à esquisser de l'un et l'autre quelques-unes des conclusions que permet une analyse sérielle des souvenirs d'élèves.

58 Le père Jean Gaudin, jésuite, grammairien poitevin né vers 1633, a notamment publié un abrégé de la grammaire latine de Despautère, citée ci-après.

59 L'expression est curieuse, Jean Despautère (c. 1460-1520) étant l'auteur de la plus fameuse grammaire latine de l'Ancien Régime, en usage jusqu'à la fin du XVIII siècle, y compris au collège de Limoges. Le mémorialiste pense-t-il que ses lecteurs l'ignorent? Ou l'a-t-il lui-même oublié?

60 Sur le collège de Limoges, cf. Marie-Madeleine Compère, Dominique Julia, Les collèges français, $16^{e}-18^{e}$ siècles. Répertoire 1 - France du midi, Paris, INRP et Éditions du CNRS, p. 365-370.

61 Cf. aussi celui de l'écrivain Florian, infra.

62 Sur ce que peut recouvrir pratiquement le terme "école", cf. Pierre Caspard, "Tenir les maîtres en bride". Les parents, la famille et l'école. France et Suisse (1700-1850)" in Jean-François Condette (dir.), Les Personnels d'inspection (XVII ${ }^{e}-X X^{e}$ siècles), Rennes, Presses universitaires de Rennes, 2017, p. 249-268. 


\section{L'apprentissage de la lecture}

Dans les parcours d'apprentissage, celui de la lecture est le plus souvent mentionné par les souvenirs. C'est qu'il fait partie de ces "premières fois " que les mémorialistes aiment à se remémorer ${ }^{63}$, entre les premiers souvenirs, les premières maladies, la première communion et les premières amours. En outre, l'évocation de cet apprentissage se prolonge souvent par celui des premières lectures ${ }^{64}$. Les trois quarts des mémorialistes, un peu moins chez les nobles, un peu plus chez les bourgeois et encore davantage chez les enfants du peuple, évoquent donc cet apprentissage premier. Par comparaison, l'apprentissage de l'écriture est deux fois moins souvent évoqué, celui du calcul ne l'est pratiquement jamais, ou sans aucune précision ${ }^{65}$.

Il ressort d'abord de ces souvenirs l'importance de l'apprentissage familial : les quatre-cinquièmes des enfants de nobles, garçons ou filles, ont appris à lire en famille. C'est le cas aussi de la moitié des enfants de bourgeois, et du tiers encore des enfants de milieux populaires. Dans les familles nobles, c'est principalement la mère qui enseigne à lire, dans les familles populaires, c'est plutôt le père. Gouvernantes ou bonnes d'enfants interviennent également dans la noblesse, jamais dans le peuple, le cas de la bourgeoisie étant intermédiaire. Enfin, d'autres parents, tantes, oncles, grand-mères, enseignent aussi à lire; dans les milieux nobles ou bourgeois, ce sont le plus souvent des femmes.

Les écoles publiques, communales ou paroissiales, jouent un rôle modeste dans l'enseignement de la lecture dont ont bénéficié les futurs mémorialistes. Aucun fils ou fille de noble n'y a appris à lire, même si le maître ou la mâ̂tresse de l'une de ces écoles ont pu être sollicités pour cet enseignement, mais en leçons particulières, "au cachet". Les enfants de bourgeois n'ont appris à lire dans des écoles publiques que pour un quart d'entre eux, le pourcentage

63 Jean-Bertrand Pontalis, psychanalyste (1924-2013), a intitulé ses souvenirs L'amour des commencements, Paris, Gallimard, 1986. Ses souvenirs d'élève y figurent p. 7-29.

64 Un exemple : Pierre Caspard, "Lectures historiques et apprentissage de l'histoire chez les filles et les garçons, 1700-1815. Une analyse sérielle de leurs souvenirs ", Genre et histoire, n²0, automne 2017, 15 p. En ligne : <https://journals.openedition.org/genrehistoire/2818>.

65 L'étude qui suit ne porte que sur une sélection non critériée de 243 souvenirs figurant dans le corpus défini supra, note 4; 180 d'entre eux évoquent un apprentissage de la lecture. Ces souvenirs portent indistinctement sur l'ensemble de la période 1490-1851, la grande majorité d'entre eux étant cependant postérieurs à 1750. Une analyse de la totalité des souvenirs du corpus permettra ultérieurement de mettre en évidence les évolutions survenues dans cette longue durée, selon les milieux sociaux et en fonction du développement du dispositif scolaire. Tous les pourcentages donnés provisoirement ici ont été arrondis. 
ne s'élevant guère qu'à un tiers pour les enfants des milieux populaires. Ce dernier chiffre éloigne de l'idée selon laquelle, depuis la Renaissance et les Réformes, les écoles publiques auraient joué un rôle essentiel et décisif dans l'alphabétisation du peuple. Pour partie, l'écart tient sans doute à ce que les mémorialistes issus du peuple appartiennent plutôt à ses franges supérieures. Mais bien d'autres sources et témoignages conduisent à relativiser, quelle que soit l'époque, le rôle des écoles publiques, au moins pour ces apprentissages rudimentaires que sont les éléments de la lecture, de l'écriture et du calcul ${ }^{66}$.

En regard, la place des "écoles particulières" est remarquable, ce terme désignant aussi bien un "maître" ou une "maîtresse" enseignant à deux ou trois enfants, à son domicile, que des écoles pouvant en accueillir plusieurs dizaines, leur point commun étant qu'elles sont dénuées de tout financement public, à la différence des écoles communales ou paroissiales ${ }^{67}$. Un enfant de noble sur cinq a appris à lire dans ce type d'école privée - c'est le cas de Chateaubriand et de sa sœur Lucile -, le pourcentage s'élevant au quart chez les enfants de bourgeois et au tiers dans les milieux populaires.

Quelques autres lieux et acteurs d'apprentissage de la lecture apparaissent d'une façon plus rare et marginale : dans une école de charité ( 1 à $2 \%$ de l'ensemble des souvenirs), dans un couvent (1\%), une poignée de mémorialistes évoquant un apprentissage plus ou moins autodidactique, ou affirmant ne plus se souvenir de la façon dont ils avaient appris à lire, trois ou quatre - dont J.-J. Rousseau - croyant "l'avoir toujours su"68.

Les souvenirs d'apprentissage de la lecture témoignent encore sur deux faits essentiels. L'un est l'âge de cet apprentissage. Il est remarquable que les deuxtiers des mémorialistes le situent entre 5 et 7 ans, quels que soient leur milieu

66 Pierre Caspard, "Le paradigme institutionnel et ses effets en histoire de l'éducation. Un exemple: les apprentissages élémentaires avant 1850 ", Histoire de l'éducation, n 144, 2015, p. 9-28. L'article souligne notamment ce qui sépare la simple acquisition des éléments de la lecture, de l'écriture et du calcul, qu'il suffira ensuite d'affermir et entretenir par la pratique, et des apprentissages plus élaborés, incluant la lecture expressive, l'écriture normée voire calligraphiée, l'orthographe, la grammaire, l'arithmétique... Les souvenirs d'élèves distinguent souvent très clairement ces étapes, et les différents maitres qu'elles peuvent mettre à contribution.

67 Sur les logiques de financement parentales, cf. Pierre Caspard, "Homo oeconomicus et ses enfants. Raisons et modalités de l'investissement éducatif dans les milieux populaires (1700-1870), in JeanFrançois Condette (dir.), Le coût des études. Modalités, acteurs et implications sociales, XVI ${ }^{e}-X X^{e}$ siècle, Rennes, Presses universitaires de Rennes, 2012, p. 171-187.

68 J.-J. Rousseau précise pourtant dans une lettre à Bernardin de Saint-Pierre qu'il aurait appris à lire à l'âge de deux ans et demi; la différence tient peut-être à ce qui sépare le fait de simplement connaitre les lettres et celui de ressentir "l'effet" produit par des lectures, en l'occurrence celle de romans, qu'il déclare avoir lus avec son père à partir de l'âge de "cinq ou six ans". 
social et le mode d'apprentissage, domestique ou collectif, public ou privé69 ${ }^{6}$ En revanche, le tiers restant recouvre majoritairement des cas de précocité, dans la noblesse et la bourgeoisie, et des cas de retard, dans les milieux populaires. Les uns et les autres sont affaire de contextes et d'opportunités, plus que d'aptitudes socialement différenciées. Les mémorialistes précisent parfois qu'ils ont appris à lire dès qu'ils en ont été capables, ou qu'ils en ont manifesté l'envie. De ce double fait, ils ne font quasiment jamais mention de difficultés qu'ils auraient pu éprouver pour apprendre à lire. Tout au plus quelques-uns gardent-ils le souvenir de maîtres ou de maîtresses ignorants et à peine capables d'enseigner (ceci valant essentiellement pour les "écoles particulières") ou brutaux et usant sans modération du fouet et de la férule (ceci valant pour les écoles publiques aussi bien que privées). De ce double fait aussi, aptitude et envie, il résulte que, à milieu social égal, les filles ont appris à lire en moyenne plusieurs mois avant les garçons, les souvenirs des uns et des autres confirmant ici ce que montrent diverses statistiques anciennes et récentes ${ }^{70}$. L'école d'État n'avait pas encore réglementairement décidé que filles et garçons devaient apprendre à lire strictement au même âge.

L'intérêt des souvenirs d'apprentissage de la lecture est aussi de l'éclairer par différents éléments de contexte. Ainsi, nombre de mémorialistes, d'origine noble ou bourgeoise, évoquent leur mise en nourrice dans quelque famille de la campagne, jusqu'à l'âge de 2,3 voire 4 ou 5 ans; certains d'entre eux précisent que, très logiquement, ils en sont revenus en parlant... le même patois que les paysans de l'endroit. Aucun d'entre eux n'évoque pourtant l'inquiétude qu'auraient pu avoir ses parents, de ce fait, sur son apprentissage de la langue française et de la lecture, même si certains, devenus Parisiens, mentionnent le mal qu'ils ont ultérieurement eu à se défaire de leur accent - provençal, picard, alsacien, basque, selon les cas.

De nombreux mémorialistes, des milieux princiers aux plus populaires, évoquent aussi les contes et histoires dont leur prime enfance a été bercée, un rapport étant implicitement, et parfois explicitement fait, entre cet

69 Ces âges sont également ceux qui apparaissent dans les autobiographies spirituelles rédigées par des puritains et des dissidents issus de milieux très populaires, dans l'Angleterre du XVII siècle : "Ils donnent l'impression d'avoir su lire couramment au plus tard à l'âge de sept ans", selon Margaret Spufford, «First steps in literacy: the reading and writing experience of the humblest seventeenthcentury spiritual autobiographers", Social History, n 4 , octobre 1979, p. 407-435.

70 Pierre Caspard, "À quoi tient la supériorité des filles? Contribution à l'analyse historique d'un problème, XVIII ${ }^{e}$-XXI ${ }^{e}$ siècles", Histoire de l'éducation, nº 115-116, 2007, p. 81-148. 
environnement oral et l'envie de l'enfant d'accéder personnellement aux livres supposés contenir ces histoires. Les mères, les servantes et les bonnes d'enfants sont le plus souvent citées dans ce rôle de conteuses, comme elles le sont d'ailleurs dans celui de maîtresses de lecture ${ }^{71}$.

Parmi les premiers souvenirs des mémorialistes figurent également les contacts physiques qu'ils ont eus avec des livres imagés, les images leur donnant envie de lire les textes d'accompagnement. Ces livres pouvaient être un almanach ou une histoire sainte, mais aussi une édition illustrée de la Bible ("Des estampes partout! Quel bonheur! Quel délire!»72), de Buffon (plusieurs fois cité) ou même, dans le cas de Chaptal, d'Aristote... pour son Histoire des animaux. Ce premier contact avec les livres résultait d'initiatives spontanées des enfants, ouvrant les livres qui leur "tombaient sous la main", selon une formule assez récurrente dans les souvenirs, plus que de stratégies pédagogiques des parents, plutôt motivés par tout moyen de tenir tranquilles leurs enfants. Les souvenirs d'apprentissage donnent peu d'exemples d'un souci parental particulier de hâter l'apprentissage de la lecture chez les enfants, quel que soit leur milieu social ${ }^{73}$. Même si la chose peut paraître culturellement implicite, les mémorialistes, qu'ils soient catholiques ou protestants, ne se souviennent pas non plus que, dans l'esprit de leur entourage, cet apprentissage aurait dû leur donner un "accès aux Écritures".

À deux ou trois exceptions près, aucun souvenir ne fait mention du rôle qu'aurait joué un précepteur dans l'apprentissage de la lecture. Le portrait de groupe que donnent des précepteurs les souvenirs de leurs anciens élèves est pourtant très circonstancié.

71 Mais trop de lectures peut tuer la lecture. Ainsi, selon les souvenirs de sa nièce, Gustave Flaubert était du matin au soir abreuvé d'histoires par sa servante, qui en connaissait un nombre infini, et par un de ses voisins, qui en connaissait tout autant. Il ne voyait donc peut-être pas la raison d'apprendre à les lire lui-même, ce qui en ferait, non "l'idiot de la famille", mais un petit malin paresseux. Cf. Matthieu Desportes, Gustave Flaubert par sa nièce, Caroline Franklin-Groult. Heures d'autrefois. Mémoires inédits. Souvenirs intimes et autres textes, Rouen, Publications de l'université de Rouen, 1999.

72 Victor Hugo, "Aux Feuillantines" (1846) dans Les Contemplations.

73 Accaparée par ses obligations mondaines à la cour de Berlin, la mère de Dorothée de Courlande, future duchesse de Dino (1793-1862) ne s'inquiète pas qu'à l'âge de sept ans, sa fille ne sache pas encore lire; c'est un baron, ami de la famille, qui s'en étonne et, après lui avoir lui-même appris les lettres, presse sa mère de la confier à une gouvernante, qui lui enseigne en huit jours à lire, prétendelle, "comme une grande personne". Mais à ce même âge de sept ans, Dorothée affirme qu' "elle parlait couramment trois langues : le français, attrapé dans le salon, l'allemand, qui m'arriva par l'antichambre et l'anglais, appris à travers les gronderies d'une vieille gouvernante" Cf. Souvenirs..., op. cit., chap. 3. C'est dire que la posture même d' "élève" n'est pas toujours requise pour certains apprentissages. 


\section{Le tout-venant des précepteurs}

À la différence des maîtres et professeurs exerçant dans des institutions scolaires, écoles ou collèges puis aussi lycées, les précepteurs sont une figure d'enseignants sur laquelle les archives publiques sont à peu près muettes. D'autres archives, familiales ou privées notamment, ont pourtant permis et permettent de tracer des portraits, individuels ou collectifs, de certains d'entre eux : ceux qui ont exercé dans des familles princières ou aristocratiques, au premier chef; les précepteurs partis enseigner le français, ou en français, à l'étranger (le mouvement inverse s'observe beaucoup plus rarement); les Huguenots du Refuge trouvant un gagne-pain dans l'enseignement; d'anciens précepteurs devenus hommes de lettres ${ }^{74}$. Ceci sans compter les textes totémiques de Montaigne, Locke, Fénelon, Rousseau ou Condillac, ni les figures de Saint-Preux ou Julien Sorel, qui saturent la mémoire historique de l'image d'un précepteur générique.

À eux tous, ils ne représentent pourtant que diverses formes d'une élite de fonction. En outre, les sources mobilisées donnent essentiellement le point de vue des précepteurs eux-mêmes, celui des parents ou de quelque observateur extérieur, mais quasiment jamais celui de leurs élèves. Par exemple, on aimerait savoir comment, en 1740, les deux jeunes enfants de Jean de Mably jugeaient le sieur Rousseau, autodidacte famélique recruté comme précepteur par recommandation d'une amie de sa maîtresse. "Je les aurais tués", confessera plus tard Jean-Jacques ${ }^{75}$; la réciproque est possible, mais on ne la connaît pas.

Tout au long de l'ancien régime scolaire, le préceptorat a été exercé, en France, par des centaines de milliers de personnes. Ceux qui sont évoqués, plus ou moins longuement, dans les souvenirs de leurs anciens élèves, nobles et bourgeois, constituent une esquisse de portrait de groupe ${ }^{76}$.

La diversité de leurs fonctions est remarquable. Les uns n'officient qu'un an ou deux, parfois quelques mois, et souvent pour enseigner le rudiment du latin à un garçon avant son entrée au collège. D'autres l'accompagnent durant

74 Cf. l'état de la question récemment dressé par Jean-Luc Le Cam, "Instruction privée et pratiques préceptorales du XVe au XIX siècle", Histoire de l'éducation, no ${ }^{\circ}$ 143, 2015, p. 9-36. Il souligne notamment notre méconnaissance du "préceptorat ordinaire".

75 J.-J. Rousseau, Confessions, livre VI.

76 Sous l'Ancien Régime, la France compte plusieurs dizaines de milliers de familles nobles et de bonne bourgeoisie. Les 362 mémorialistes de sexe masculin, de famille noble ou bourgeoise, figurant dans le corpus défini supra, évoquent le souvenir de l'enseignement qu'ils ont reçu de plus de deux cents précepteurs, une majorité d'entre eux étant en fonction au XVIII ${ }^{e}$ siècle. 
toute son enfance et son adolescence, parfois jusqu'à l'université, en lui servant à la fois de gouverneur et de mentor. Certains s'occupent de tous les enfants d'une même famille, d'autres sont attachés à chacun d'entre eux, quel que soit leur nombre. La majorité sont des jeunes gens tout juste sortis de quelque collège ou séminaire, dans l'attente d'une position plus stable, en comptant éventuellement sur l'appui du père de famille. Les deux-tiers de ceux dont l'âge est mentionné ont entre 17 et 24 ans; certains d'entre eux sont des émules, plus que des mentors. Le terme d'abbé, qui les désigne fréquemment, signifie qu'ils ont été tonsurés, sans plus, mais ne préjuge pas d'un engagement religieux particulier, ni même, au XVIII e siècle en tout cas, d'une simple croyance en Dieu. D'autres sont des hommes âgés ou des vieillards, monnayant leurs soins par le gîte et le couvert. Entre les têtes bien faites, les têtes bien pleines et celles qui ne sont ni l'un ni l'autre, la diversité de leurs compétences n'est pas moindre que celle de leurs fonctions, et suscite chez les mémorialistes une large gamme d'appréciations. Elle va du précepteur ignorant, brutal, paresseux, stupide, ivrogne, hypocrite, laid, dépourvu de méthode, trop tendre ou au contraire sénile, jusqu'au précepteur savant, doux, dévoué, amical, cultivé, bon pédagogue, connaissant les usages du monde, en passant par les pédants, les cuistres, les bigots, les mécréants, les coureurs de filles ou, quelquefois, de garçons. Naturellement, les précepteurs pouvaient combiner plusieurs de ces qualités et/ou défauts. Voici le souvenir qu'a des siens l'écrivain Florian, dans un chapitre de ses mémoires précisément intitulé "Mes précepteurs" ${ }^{77}$.

Né en 1755 à Sauve (2800 habitants en 1793), dans le Gard, Jean-Pierre Claris de Florian a perdu sa mère très jeune. Son père, officier, est le plus souvent absent. Il est élevé par sa tante. De quatre à sept ans, il apprend à lire et à écrire à Saint-Hippolyte (5000 habitants en 1793) "chez une demoiselle qui tenait des pensionnaires" ; puis, de sept à huit ans, "dans une espèce de collège" où il "se perfectionne dans la lecture et dans l'écriture, sans rien apprendre au-delà". Après une année pour ainsi dire sabbatique, consacrée à la chasse et à la lecture passionnée de L'Iliade, il se rend à l'âge de dix ans à Paris, où il reçoit l'enseignement de précepteurs.

Le premier qu'on lui donne est un homme de cinquante ans, "né avec de l'esprit et beaucoup de connaissances" [sic], mais qui s'intéresse moins à son élève qu'à l'écriture d'une tragédie, Les Chérusques, "dont le succès [ultérieur]

77 Mémoires d'un jeune Espagnol, de Florian, Paris, Librairie des bibliophiles, 1883. 
semble prouver qu'il n'était pas sans talent». Le second est "un incapable qui ne sait pas un mot de latin". Rapidement congédié, il est suivi par un troisième, un abbé "dont la science était la seule qualité", car il est oisif et d'une extrême brutalité. Renvoyé, il est remplacé par un quatrième précepteur, encore un abbé, "qui ne sait que médiocrement le latin"; en outre, il s'adonne à la boisson, et va fréquemment rendre visite à une demoiselle avec son élève, laissant cependant ce dernier dans l'antichambre en compagnie d'un gros chat, pendant qu'il a des entretiens particuliers avec la demoiselle. Enfin, le cinquième et dernier précepteur qu'il reçoit, à l'âge de douze ans, est "un homme bien au-dessus de son état, plein d'esprit et d'érudition, de mœurs irréprochables et fait, en un mot, pour rendre son disciple vertueux, aimable et instruit". Il le conserve jusqu'à l'âge de treize ans, quand il se rend à Versailles continuer son instruction ${ }^{78}$.

Les souvenirs de Florian témoignent de deux problèmes assez récurrents pour les familles. L'un est leur manque de repères, dans un marché préceptoral dépourvu de toutes certifications. Les recommandations jouent un grand rôle (cf. J.-J. Rousseau), mais rares sont les précepteurs qui enchaînent assez d'expériences pédagogiques pour asseoir une réputation ou même se prévaloir d'une méthode d'enseignement un peu éprouvée. Recruter un précepteur tient donc de la loterie, dont les souvenirs du prince Charles-Joseph de Ligne (1735-1814) ou de Benjamin Constant (1767-1830), tous deux fils d'officiers, l'un vaudois, l'autre wallon, donnent d'autres exemples très significatifs ${ }^{79}$. La remarque vaudrait aussi pour les gouvernantes, nurses et bonnes d'enfants, même si leurs fonctions éducatives ou enseignantes sont généralement évoquées avec beaucoup moins de précisions que celles des précepteurs. Joseph Primoli déclare ainsi "avoir vu défiler jusqu'à quatorze Miss chargées de lui apprendre la langue de Shakespeare"; l'une le bat, une autre boit, une troisième vole,

78 À Versailles, il reçoit une formation de page pendant deux ans, sous la direction d'un abbé "petit, laid, méchant, ignorant, sot et tartuffe ". À quinze ans, le désir le prend "tout à coup" de servir dans l'Artillerie; il travaille jour et nuit les mathématiques avec un maitre parisien et, à seize ans, il est reçu au concours d'entrée à l'école royale d'Artillerie de Bapaume. Il sera plus tard l'auteur de fables et de nouvelles, ainsi que du texte de l'immortel "Plaisir d'amour".

79 Entre neuf et seize ans, Charles Joseph se voit confié successivement à neuf précepteurs : un exjésuite, deux pages, un abbé, un ex-jésuite régent du collège Louis-le-Grand, un officier de cavalerie, un ex-oratorien, un capitaine des hussards et encore un ex-jésuite du collège Louis-le-Grand. Cf. Mémoires, lettres et pensées ..., op. cit., p. 45-51. Entre cinq et quatorze ans, Benjamin Constant en connait successivement six : un "Allemand" (helléniste? Il commence à lui apprendre à écrire en lettres grecques à cinq ans...), un chirurgien-major, un maitre de musique, un ex-avocat, un moine français défroqué, un jeune Anglais et un pasteur suisse de vingt-quatre ans. Cf. Le Cahier rouge, in CEuvres, Paris, Gallimard, 1984, p. 87-90. 
une quatrième est folle, une cinquième est étourdie au point de perdre son frère dans les rues... Deux seulement ont été "supportables" : " une vieille fille pauvre, presque religieuse", et "une excellente fille, très vive et très enfant "

Le second problème est celui de la capacité des parents à juger de la qualité du travail du précepteur. Dans le cas de Florian, sa tante apparaît très réactive : l'incapable, la brute et l'ivrogne sont vite congédiés. Mais dans bien d'autres cas, les parents, le père en particulier, sont trop occupés, voire absents, pour suivre le travail du précepteur ou pas toujours assez compétents pour l'évaluer : c'est le cas des pères du prince de Ligne et de B. Constant. Plus rares sont les pères qui exercent un contrôle strict, remettant au précepteur un plan de travail, exigeant qu'il tienne un journal des progrès de son élève, demandant à consulter ses productions écrites, compositions ou "extraits", ou échangeant avec lui une correspondance suivie, s’ils sont durablement éloignés ${ }^{81}$.

Le plus souvent, les souvenirs d'anciens élèves de précepteurs ne sont pas rapportés pour nourrir le débat, particulièrement récurrent au XVIII ${ }^{\mathrm{e}}$ siècle, sur les mérites respectifs des enseignements public et domestique. L'observation vaudrait un peu moins pour les souvenirs de l'enseignement reçu au collège ${ }^{82}$. Il s'agit plutôt d'évoquer, comme telles et parmi d'autres, les expériences humaines et pédagogiques, bonnes ou mauvaises, qui ont jalonné l'enfance et l'adolescence des mémorialistes. Ceci donne une allure assez vraisemblable au portrait collectif qu'ils permettent de dresser de ce que l'un d'eux appelle drôlement «le corps préceptoral " ${ }^{83}$, alors même qu'au contraire du corps enseignant en voie

80 Giuseppe Primoli, Mémoires..., op. cit., p. 499-500.

81 Cf. la correspondance échangée de 1769 à 1783 entre un bourgeois de Lille, greffier des États de la Flandre wallonne, et le précepteur de ses trois garçons, d'abord à Lille même, puis à Paris lorsqu'ils sont scolarisés aux collèges de Lisieux puis de Navarre : Philippe Marchand, Donnez-moi des nouvelles... Collèges et collégiens à travers les correspondances familiales, 1767-1787, Lille, Presses universitaires du Septentrion et Archives du Nord, 2018.

82 Lucien Bély, "L'élève et le monde, essai sur l'éducation des Lumières, d'après les mémoires autobiographiques du temps", Revue d'histoire moderne et contemporaine, janvier 1981, p.3-35.

83 Vieux souvenirs de Mgr le prince de Joinville, 1818-1848, Paris, Mercure de France, 1970. François d'Orléans (1818-1900) est le septième enfant du futur roi Louis-Philippe; sa mère, MarieAmélie de Bourbon, lui apprend à lire puis, à six ans, il est confié à un précepteur (un distingué normalien), comme chacun de ses cinq frères, ses trois sœurs ayant de leur côté deux gouvernantes. C'est cet ensemble qu'il qualifie avec humour de "corps préceptoral", en décrivant le fonctionnement quotidien du collectif des huit adultes avec leurs neuf élèves. À dix ans, il entre au collège Henri-IV, où il avoue avoir été "un cancre, rien qu'un cancre", expression ancienne pour désigner ce qu'il est convenu aujourd'hui d'appeler une "victime de l'échec scolaire". 
de gestation depuis l'Ancien Régime ${ }^{84}$, ce "corps" est ouvert aux quatre vents de l'offre et de la demande.

Identifiés dans des textes au genre incertain, constitués en corpus et analysés dans une longue durée, les souvenirs d'élèves peuvent contribuer à une histoire de l'éducation compréhensive, c'est-à-dire attentive aux raisons, moyens et manières d'apprendre et d'enseigner de l'ensemble des acteurs impliqués, qu'ils soient familiaux, institutionnels ou marchands. Ceci vaut pour l'ancien régime scolaire, où les souvenirs d'élèves sont assez nombreux pour se prêter à des analyses sérielles de parcours et d'expériences, sans que ceux-ci soient encore pré-formatés au sein d'un système scolaire global. Une autre question serait d'identifier, en aval d'une plus longue durée, ce qui subsiste de ces logiques et comportements ou s'y substitue, une fois le système mis en place ${ }^{85}$.

Pierre Caspard

Directeur de recherche honoraire

p.caspard@gmail.com

84 Boris Noguès, Une archéologie du corps enseignant. Les professeurs des collèges parisiens aux XVII et XVIII ${ }^{e}$ siècles (1598-1793), Paris, Belin, 2006.

85 Ainsi, Jean-Michel Chapoulie a analysé la part inédite que peut prendre désormais, dans le cas des parcours d'élèves scolarisés dans les écoles primaires supérieures de la première moitié du $\mathrm{XX}$ siècle, "l'abandon aux initiatives de l'institution scolaire et de ses agents". Cf. "Sur les rapports des classes populaires et moyennes à l'école (France, 1910-1950) : quelle "demande sociale" d'instruction prolongée?", Revue d'histoire moderne et contemporaine, $\mathrm{n}^{\circ}$ 3, 2018, p. 59-93. 